TM-1599

\title{
Fermilab Fixed Target Beams from the Main Injector*
}

S. Childress, R. Coleman, G. Koizumi, A. Malensek, C. Moore, R. Schailey, R. Stefanski, and L. Stutte

Fermi National Accelerator Laboratory

P.O. Box 500, Batavia, Illinois

May 1989

* Reference work for the Main Injector Workshop, Fermilab, May 16-18, 1989.

Operated by Universities Research Association, Inc., under contract with the United States Department of Energy 


\title{
FERMILAB FIXED TARGET BEAMS from the MAIN INJECTOR
}

\author{
S. Childress \\ R. Coleman \\ G. Koizumi \\ A. Malensek \\ C. Moore \\ R. Schailey \\ R. Stefanski \\ L. Stutte
}

Fermi National Accelerator Laboratory

P.O.Box 500, Batavia, Illinois 60510

May 1989 


\section{Fermilab Fixed Target Beams from the Main Injector}

Contents

Page

1. Overview : 120-150 GeV Beams 1

2. 120-150 GeV Extraction

2.1 Resonant Extraction

2.2 Debunched Beam in the Main Injector

3. Link to Switchyard

11

4. Primary Beam Splits and Transport

4.1 Test Beams to All Experimental Areas

14

4.2 New High Intensity Beam Line to Neutrino Area

25

5. Experimental Facilities at $\mathbf{1 2 0 - 1 5 0} \mathrm{GeV}$

5.1 A Model for High Intensity Facilities

5.2 High Sensitivity Kaon Experiment

5.3 Neutrino Oscillation Experiment

45

5.4 General Purpose Neutrino Beam

48

5.5 Focusing Systems

50

5.6 Target and Dump Cooling Requirements 


\section{FERMILAB FIXED TARGET BEAMS from the MAIN INJECTOR}

\section{Overview : 120-150 GeV Beams}

This report details possible models for enhancements to the Fermilab fixed target beams system, utilizing the capabilities of the proposed new Main Injector. Projections for new fixed target beam line capabilities are grouped into two major categories:

An extraction system from the Main Injector, linking to the existing Switchyard, and providing $120 \mathrm{GeV}$ test beams to all major experimental areas during collider running.

A high luminosity $120-150 \mathrm{GeV}$ beam line to the Neutrino area for a new generation of high flux experiments; utilizing the unique combination of beam intensity, repetition rate, and energy made available with the Main Injector.

The first section of the report discusses details of extracting beams from the Main Injector. These beams are then linked to the existing Switchyard system to provide test beam capability to all fixed target experimental areas during collider running periods. Fixed target beam capability, even at low duty cycle, during collider running should greatly enhance preparation for efficient physics runs. Additionally, improvements in detector function may be realizable which would otherwise not be possible without the option of significant amounts of beam study time. 
A high intensity fast repetition rate dedicated $120-150 \mathrm{GeV}$ beam line to the Neutrino area is proposed, enabling the running of experiments for which high luminosity is more critical than Tevatron beam energies. At least two such experiments are being seriously considered by Fermilab Users at present. The first is a very high sensitivity Kaon experiment, reaching branching ratio sensitivity to rare decays at the level of $10^{-10}$ per hour. This experiment would require debunched extracted beams from the Main Injector. The second type of experiment considered is a neutrino oscillation experiment, which would require high luminosity fast resonant extracted beams.

Additionally, with the Main Injector, the capability for a year round high luminosity 120 - $150 \mathrm{GeV}$ fixed target physics program would be present. This program would be compatible with, and could run simultaneously with, both the existing Tevatron collider and fixed target HEP programs. 


\section{$2 \quad 120-150$ GeV Extraction}

The Main Injector provides greatly enhanced fixed target capabilities beyond what might be achieved by implementing an extraction system from the existing Main Ring. Additionally, major problems of a fundamental nature encountered with a Main Ring extraction system are avoided.

Some of the Main Ring problems which make a compatible fixed target extraction system potentially very difficult are: limited horizontal aperture; severe limits on allowable extraction beam loss imposed by the Collider detectors; and spatial conflicts at $\mathrm{A} 0$ due to the $8 \mathrm{GeV}$ injection line, Tevatron extraction, and Collider aborts.

These problems are largely eliminated with the Main Injector.

\subsection{Resonant Extraction from the Main Injector}

A Main Injector extraction system has been designed to provide $120 \mathrm{GeV} / \mathrm{c}$ resonant extracted beam with a uniform slow spill of one second, and $150 \mathrm{GeV} / \mathrm{c}$ fast resonant extracted beam with spill duration of $2 \mathrm{~ms}$. Extraction losses of less than $3 \%$ should be achieved. By comparison, Tevatron resonant extraction losses are consistently less than $2 \%$, with the improvement primarily due to high beta modification to the Tevatron lattice. This has not yet been modeled for the Main Injector, but could possibly produce similar improvement in extraction beam loss. Slow spill of the entire beam is easily achieved with half-integer extraction.

Extraction is implemented by using special quadrupoles and octopoles to bring the beam onto the half-integer resonance in such a manner that the amplitude of betatron oscillations will grow in a controlled fashion until the particles are deflected by an electrostatic septum. The kick supplied by the electrostatic septum provides enough space between the circulating beam and the extracted beam to allow magnetic septa to be used to extract the beam. 


\section{Septa}

The choice of the location of the magnetic septa (Lambertson magnets) is dictated by the location of the extraction channel to the A0 area of the Tevatron. The Lambertsons will be placed in the upstream region of the MI-60 straight section in order to maximize the transverse separation when the extracted beam reaches the location of the downstream quadrupoles. The expected separation of $31 \mathrm{~cm}$ generated over $19 \mathrm{~m}$ by two standard $5 \mathrm{~m}$ Lambertsons running with a field of $9 \mathrm{kG}$ will be adequate to miss the first quadrupole.

The placement of the electrostatic septum is made easy by the fact that a location $90^{\circ}$ in phase is available in the cell immediately upstream of the long straight section. The slot length is adequate for a septum of $3.6 \mathrm{~m}$. It is desirable to have a separation of $6 \mathrm{~mm}$ at the magnetic septa between the circulating beam and the extracted beam. A $120 \mu \mathrm{R}$ kick from the electrostatic septum will achieve this, and with the length available, the applied voltage gradient of 40 $\mathrm{kV} / \mathrm{cm}$ will not be excessive.

\section{Harmonic Elements}

The appropriate harmonics for the quadrupoles (45th) and octopole (0th) will be provided by special magnets designed for this purpose. The quadrupoles will be located at points equidistant around the ring (in phase). Air core quadrupoles are sufficient to provide the necessary stop band width to ensure total extraction of the beam. The calculated integral strength of the quads is $60 \mathrm{kG}$-in @ 1 inch, and for the octopoles 116 kG-in @ 1 inch.

\section{Modeling Program}

The technique used to model resonant extraction is a matrix multiplication program which takes a distribution of particles in phase space and propagates it around the ring. In the approximation that the lattice parameters do not change with small changes in tune, then the tune of the ring can be adjusted from the nominal operating point of 22.42. The ring can be broken at any desired location and extra elements added; additionally the particle distribution can be 
displayed at any point. In general the displays shown are at a given position in the lattice and show the turn by turn behavior of the particles. In order to cut down computer disk space requirements not every turn is displayed; however at the septa locations, every extracted particle is shown on its final turn thus defining the extracted beam phase space. The distribution in phase space is derived from the assumed emittance. In fact, the distribution used is uniformly distributed in phase space; the reason for this is to conserve computer time in examining all of phase space for problems. The extraction dynamics are relatively insensitive to the initial phase space distributions.

\section{Phase Space}

The phase space variables used to display the data are: the position of the particle on the horizontal axis, and on the vertical axis the conjugate variable which makes the unperturbed motion of a particle a simple circle in normalized phase space. If the position is $x$ and the angle is $x^{\prime}$, then this conjugate variable is $y=$ beta* $x^{\prime}+$ alpha* $x$ where alpha and beta are the normal Courant-Snyder variables, and the Courant-Snyder invariant is $W=\left(x^{2}+y^{2}\right) /$ beta. When the particle has physically traversed the ring once, it will have made $Q(\sim 22.5)$ revolutions around the phase space circle. The consequence is that when we have a distorted phase portrait at a point in the ring as happens in extraction, we know the qualitative shape of the distribution at any other point in the ring: the pattern is rotated by the betatron phase difference (modulo 360 degrees), and the relative scale difference is given by the ratio of the square root of the betas.

Extraction starts by raising the normal horizontal tune from 22.42 towards 22.5 , while changing the zeroth harmonic octopole component of the ring. One uses the octopole to produce an amplitude dependent tune shift; particles with a large amplitude having a larger tune, i.e. closer to the half-integer. Then a 45th harmonic quadrupole set is used to increase the half-integer stop band. Small amplitude (smaller tune) particles are stable while larger amplitude particles stream out along the separatrix until they encounter the electrostatic septum. 
This process is sketched below:

Stop band

width

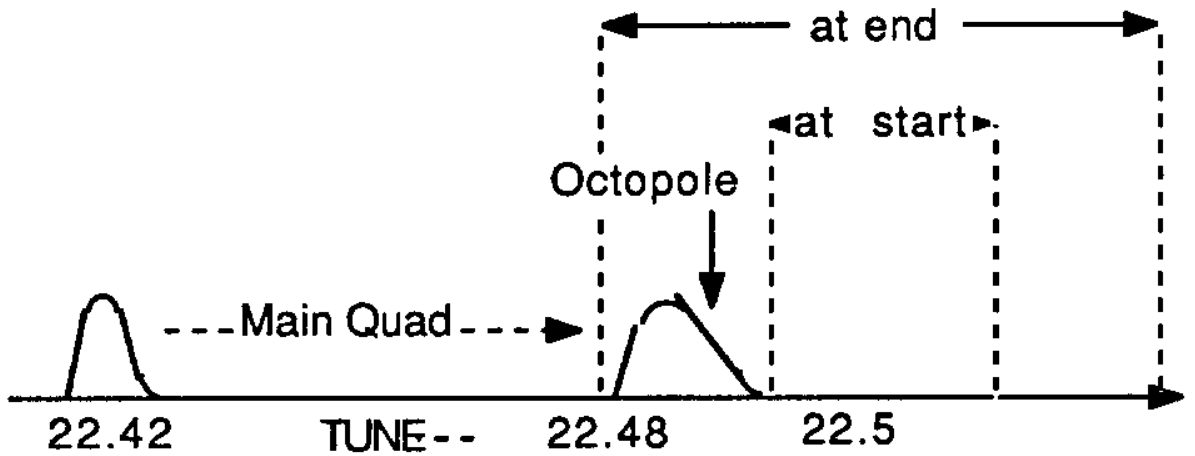

Phase space portraits produced by these elements at the electrostatic septum and at the magnetic septa are shown in Figure 2.1. As indicated by the discontinuity in the arms the effect of the septum is modeled by a change in $x^{\prime}$, with no change in $x$. The separatrix phase of 45 degrees exhibited by the beam is a compromise between high extraction efficiency (where the angle would be zero) and aperture considerations at the location of the magnetic septa (i.e. if the angle were zero at the electrostatic septum, the magnetic septa would be in the center of the aperture). Also we see that there is sufficient displacement between the circulating beam and the extracted beam, and both kinds of septum are reasonably far removed from the center of the aperture. For the extracted phase space shown, the emittance is $6 \pi$ with an extraction inefficiency of $3 \%$.

\section{OXR}

The goal of the slow extraction system is to provide a constant rate of extraction during the course of the slow spill. This is done by moving the stop band smoothly through the beam, using a system called QXR (quad extraction regulator). There are two parts to this system, separated by strength and bandwidth. The stronger lower bandwidth components are tied to a normal beam intensity monitor which is not sensitive to fast fluctuations. After the signal is sampled during the spill and compared to an ideal signal, the resultant smoothed error signal is used to modify the power supply output. This system is the base from which the weaker faster responding system works. Its monitoring is based upon a fast reacting detector 
in the extracted beam line itself. An example of the type of error signal that this component can handle is power supply ripple up to about $360 \mathrm{~Hz}$. (Identical to that found in the Tevatron). 


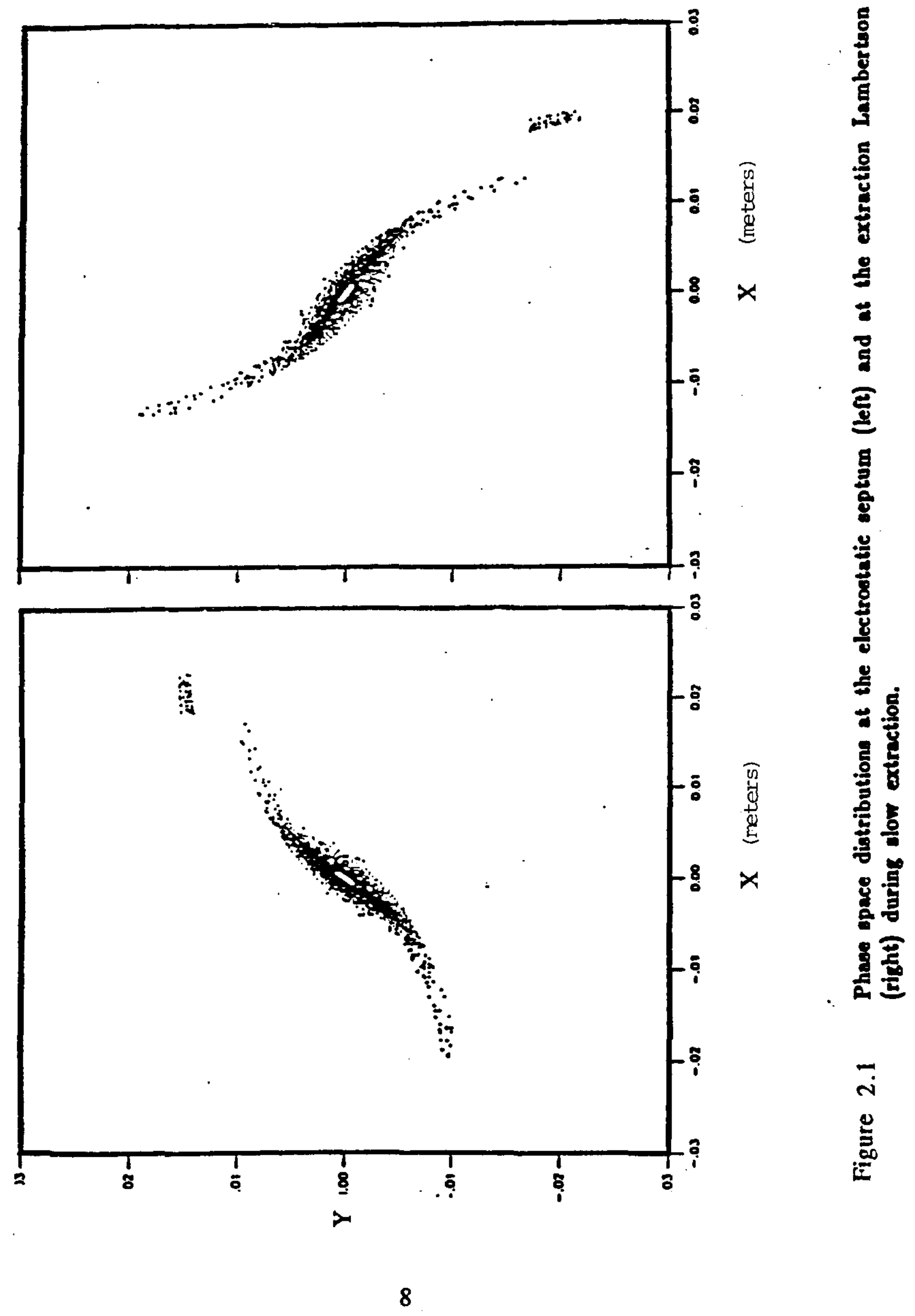




\subsection{Debunched Beam in the Main Injector}

Debunching the beam in the Main Injector does not appear to present great technical difficulty. The R.F. cavities will be the present Main Ring cavities and the rest of the Main Injector will be a much smoother object than the Main Ring. Hence, our experience with beam loading of the cavities will be immediately transferable and the overall longitudinal impedance will be much smaller.

There are various scenarios that could be followed to produce debunched beam. We can divide them into two general methods that differ in how quickly the R.F. is turned off. The first method is to quickly reduce the R.F. voltage, letting the bunches shear at a large $\mathrm{dp} / \mathrm{p}$. This method has a quick debunching time in terms of a uniform distribution of charge. A disadvantage is that beam loading on the cavities would tend to rebunch the beam immediately after the R.F. is switched off. However, beam loading is easy to compensate for a full ring and the compensation can be made to track the intensity remaining in the ring. The second method is to adiabatically reduce the R.F. voltage by first paraphasing stations, then turning stations off, inserting cavity shorts, and finally paraphasing to zero voltage with two R.F. stations. Using this method the beam loading effects are greatly reduced at the expense of a longer charge debunching time.

The question of the uniformity of the longitudinal charge distribution is not the only question, since slow extraction can be influenced by the momentum spread in the beam via chromaticity. However, for beam stability one need only have slightly positive chromaticity, and as long as the tune spread induced by chromaticity is small compared to the tune spread induced by the octopoles then the $53 \mathrm{Mhz}$ structure in the momentum distribution will not propagate into the spill structure. One advantage the adiabatic methods have is that they reduce the total momentum spread in the beam before turning off the R.F., and hence the possible effects from momentum spread are reduced. 
There are various gradations and combinations of the two general scenarios. In fact one could even think of blowing up the $\mathrm{dp} / \mathrm{p}$ of the bunch as we do for pbar targeting; this would yield an even quicker debunching time. However, a reasonable scenario might be to simply turn the R.F. off at $4 \mathrm{MV}$, use active beam loading compensation, turn the chromaticity down, and while the octopoles are ramping up, simply allow the bunches to shear out over 8 adjacent buckets ( $\sim 50 \mathrm{~ms}$ is required) before starting to slow extract.

The microwave instability which is induced by the longitudinal impedance of the rest of the ring should not cause a problem, since the longitudinal impedance of the Main Injector will be much less than the impedance of the Main Ring. Coalescing experience of the $2 b$ cycles with $2.4 \times 10^{10}$ per bunch has shown no problem, and in this case the R.F. is adiabatically reduced to almost zero.

The conceptually simple method of turning off the R.F., and waiting $50 \mathrm{~ms}$ will produce bunches which have spread out over eight buckets. With a small enough chromaticity, this method should produce a spill uniformity of much better than 2 to 1 at the beginning of extraction. Also, one should realize that this will be one instance where things get better; i.e. the spill will be the most uniform at the end of extraction when the most spreading out has occurred. 


\section{Link to Switchyard}

Constraints for connection to the Switchyard beam line system are much simpler for extraction from the new Main Injector than would be required for extracting from the existing Main Ring. A system for Main Ring extraction at A0 would be required to maintain compatibility with the existing Tevatron extraction line at the same location; the redesigned $8 \mathrm{GeV}$ Main Ring injection line; and the new Collider abort system, to be installed at A0 before the FY91 Collider running period.

The new Main Injector considerably simplifies extraction conflicts at $\mathrm{A} 0$, and also enables simultaneous (alternate pulse) capability of the Switchyard test beams and dedicated high intensity beam line during collider running periods. The dedicated beam line would additionally be fully compatible with Tevatron fixed target running to the Proton, Meson and Muon areas; thus being available for year round use.

Linking of the extracted Main Injector beam to the existing Tevatron Switchyard is established in a straightforward manner, with only minimal changes required at the Switchyard interface.

Design of the beam line transport from the Main Injector to A0 is discussed in the Main Injector Conceptual Design Report. At A0, the extracted $120-150 \mathrm{GeV}$ Main Injector beam is sufficiently far removed to the West from the Tevatron extraction system that interference is minimal.

Beam from the Main Injector is switched between the test beam channel linked to the existing Switchyard system and the dedicated high intensity line by changing currents in a magnetic switch located in the A0 straight, upstream of existing Switchyard beamline elements. Switching of Main Injector beam into either channel can be readily accomplished at any time, with no repositioning of magnets required. 
Final positioning of the test beam link from the Main Injector onto the Tevatron Switchyard trajectory is accomplished with a vertical bending dipole to be located upstream of PSEP. Space for this dipole would be obtained by shifting the PSEPs downstream by 12.6 $\mathrm{ft}$,, the length of one septa module. This results in a small but inconsequential loss in splitting effectiveness of the PSEP string. The new dipole is then energized to link the Switchyard system with the Main Injector test beam line, and is turned off to maintain the existing Tevatron extraction line.

Since the test beam link intersects the existing Switchyard channel upstream of the first electrostatic septa station (PSEP), test beam splits to all fixed target experimental areas are feasible.

The vertical size of the $150 \mathrm{GeV}$ beam is considerably larger than that of the Tevatron extracted beam, when using existing optics, as is detailed in Section 4.1. A solution which enables the Main Injector beam to fit through the narrow PSEP aperture requires a quadrupole shifted upstream a considerable distance from the first existing Switchyard quad, Q90 centered at $Z=141 \mathrm{ft}$. This is best accomplished with a new $3 \mathrm{ft}$. quad in the Main Injector extraction channel centered at $\mathrm{Z}=51.5 \mathrm{ft}$.

Clearances for the Main Injector extracted beam relative to existing elements at the downstream end of the $\mathrm{AO}$ long straight section are shown in Figure 3.1. The present Main Ring quad at A11 is maintained in the figure to indicate compatibility of the Main Injector link to Switchyard with a possible new ring in the $\mathrm{TeV}$ tunnel, replacing the existing Main Ring. The extracted test beam is centered more than 2.5 inches from edges of the last Tevatron extraction channel skew dipole and an upper ring magnet at A11 comparable in cross section to a Main Ring quad; a generous clearance by Switchyard norms. 


\section{Beam Line Clearance at A11 Extracted Main Injector Beams}

$$
\mathrm{Z}=\mathbf{8 3 . 4} \mathrm{Ft}
$$

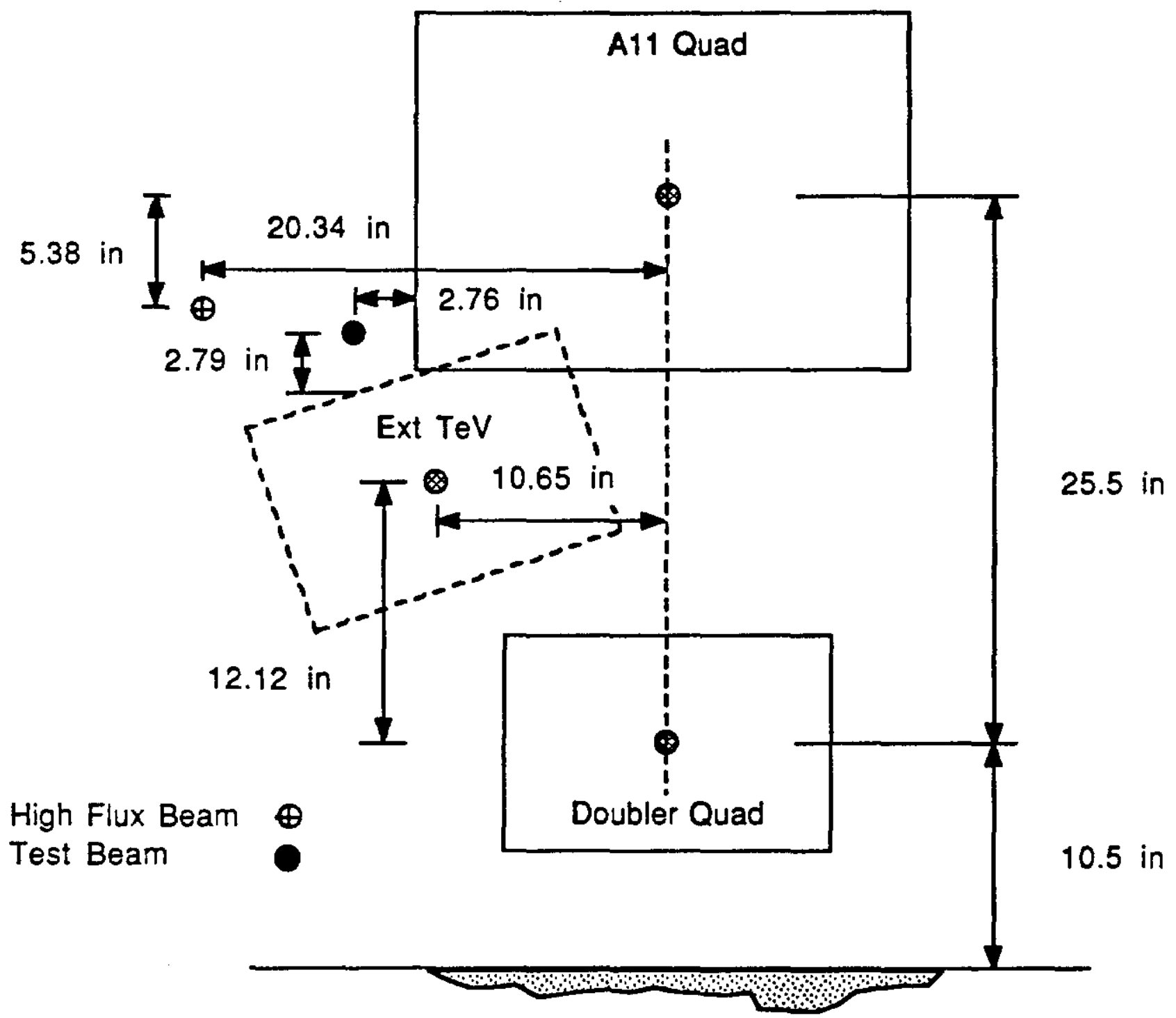

Figure 3.1 


\section{$4 \quad$ Primary Beam Splits and Transport}

For test beam capability to all experimental areas the 120 $150 \mathrm{GeV}$ beam from the Main Injector must be split and transported through the existing Switchyard beam lines. A major concern is aperture limitations due to the larger size of the reduced energy beam, and this has been studied in some detail. All present Switchyard lines work well for $400-800 \mathrm{GeV}$ beams, enabling any desired beam fraction to be transmitted to either side of each septa splitting station. A worst case fall back position for $120-150 \mathrm{GeV}$ would involve giving up flexibility of turning each experimental area test beam on and off independently; at present, however, it does not appear that this will be required.

The new dedicated High Intensity line to the Neutrino area does not have to be transmitted through the narrow aperture septa splitting stations; thus magnet aperture versus beam size is not as serious a concern. The challenge for this line is being able to stage a new independent beam line in the existing Switchyard tunnel enclosures, with a minimum of civil construction costs.

\subsection{Test Beams to All Experimental Areas}

After linking the extracted beam from the Main Injector to the upstream of the Switchyard beamline system, $120-150 \mathrm{GeV}$ low intensity test beams should be able to be transmitted to the Fixed Target experimental areas during Collider physics runs. The major concerns after beam extraction are aperture and optics considerations due to significantly larger beam emittance at the lower energy. 


\section{Emittance Assumptions}

The emittance for a $150 \mathrm{GeV}$ beam will be different from that currently observed in the $800 \mathrm{GeV}$ fixed target program. Since the horizontal emittance is primarily determined by the extraction process, it is assumed that the $150 \mathrm{GeV}$ horizontal beam size and divergence are the same as observed in the last Fixed Target run. Since the vertical emittance should scale as $1 / \mathrm{p}$, we will assume that both the vertical beam size and divergence are given by $1 / \sqrt{\mathrm{p}}$ times those observed in the last run. Cross terms will be assumed to be unchanged.

SWIC pictures of extracted beam profiles throughout Switchyard and in pre-target locations in the Experimental Areas, obtained near the end of the 1987-88 fixed target running period, have been used to determine the $800 \mathrm{GeV}$ emittance. In both the horizontal and vertical planes, the SWIC profiles were measured to determine their full width at half maximum. These beam sizes were then entered into TRANSPORT as constraints, equating them to halfwidth sizes at the base, as used in this program. The emittance parameters of the beam were then fit to best match the beam profiles as observed. Separate fits were done for each of the experimental areas: Proton, Neutrino and Meson. The Neutrino and Meson fits were very similar in all 6 parameters varied. The Proton fit was quite different. To date, this discrepancy has not been resolved: using the Proton fit results in Neutrino or Meson (and viceversa), causes the beam envelope to blow up to several times the beam sizes observed. For the purpose of this study, however, in the interest of simplicity, the emittance determined from the Neutrino fit has been scaled to $150 \mathrm{GeV}$ as described above. 
The parameters used for this optics study are as follows:

$\begin{array}{lcl}\mathrm{x}: & 0.20963 & \mathrm{~cm} \\ \Theta_{\mathrm{x}}: & 0.02439 & \mathrm{mrad} \\ \mathrm{y}: & 0.26331 & \mathrm{~cm} \\ \Theta_{\mathrm{y}}: & 0.12443 & \mathrm{mrad} \\ \mathrm{dx} / \mathrm{d} \Theta_{\mathrm{x}}: & -0.57341 & \mathrm{~cm} / \mathrm{mrad} \\ \mathrm{dy} / \mathrm{d} \Theta_{\mathrm{y}}: & 0.68640 & \mathrm{~cm} / \mathrm{mrad}\end{array}$

\section{Aperture Limitations}

The most restrictive apertures encountered in Switchyard are those found at the electrostatic septa, which have $2 \mathrm{~cm}$ wire to cathode gaps. For the most flexible splitting arrangements, this implies that the beam should be less than $1 \mathrm{~cm}$ half-width in these locations. The next most restrictive apertures are those in the Lambertson magnets, which have a $3.8 \mathrm{~cm}$ diameter field-free hole separated by 150 mils from a field region, which is $2.8 \mathrm{~cm}$ wide in the non-bend dimension by $12.7 \mathrm{~cm}$ wide in the bend dimension. Energy Saver dipoles provide apertures of $7.1 \mathrm{~cm}$ diameter. All remaining magnets in the Switchyard are taken to be EPB dipoles $(12.7 \mathrm{~cm}$ by $3.6 \mathrm{~cm})$, EPB quads $(7.6 \mathrm{~cm}$ diameter), and trims $(10.2$ $\mathrm{cm}$ by $5.1 \mathrm{~cm})$.

\section{New Upstream Ouadrupole}

Because of the larger emittance at $150 \mathrm{GeV}$, it is no longer possible to fit the beam through the first septa (PSEP) encountered after extraction, with existing beam optics. One option requires a second parallel Switchyard $150 \mathrm{GeV}$ beam channel through the PSEP split. With this option it becomes possible to add a new quadrupole upstream of the PSEP's, in the region just downstream of the skewed dipoles. This quad (designated QSK), is five feet in length, and runs with a $-1.69 \mathrm{KG} / \mathrm{in}$ gradient. This quad then allows the beam to fit through the first septa string. 
As a consequence of using this new quadrupole, the next quad encountered, Q90 (the first used for $800 \mathrm{GeV}$ optics), is not used.

The other option would attempt to fit a very short new quad in the downstream portion of the $150 \mathrm{GeV}$ extraction line, significantly before the intersection point with the existing Switchyard upstream of the PSEP split. Feasibility of this is still under evaluation, but now appears viable, as was discussed in Section 3. If feasible, this is the preferred solution.

Optics calculations in this section assume a quad QSK just downstream of the skewed $\mathrm{TeV}$ extraction dipoles. An alternative quad placement further upstream would produce a smaller vertical beam size at PSEP than shown here.

\section{Additional Downstream Ouadrupoles}

Again, because of the increased emittance of the $150 \mathrm{GeV}$ beam, it is necessary to add a few additional quadrupoles in downstream locations in order to fit the beam through restrictive apertures. In the Neutrino area, a single quad in Enclosure C, just upstream of the MUSEP's is sufficient. This quad, called Q106, is 5 feet in length, and runs at a gradient of $-0.38 \mathrm{KG} / \mathrm{in}$. In both the Proton and Meson areas, quadrupole doublet systems are needed to contain the $150 \mathrm{GeV}$ beam. These quads are located downstream of the ESEP's and the FSEP's, respectively; similar in placement and function to the quads Q120 and Q121 in G2, which follow the NSEP's. The Proton quads are designated Q307 and Q308, and run at gradients of $0.87 \mathrm{KG} / \mathrm{in}$ and $-1.22 \mathrm{KG} / \mathrm{in}$, respectively. The Meson quads are designated $\mathrm{Q} 220$ and $\mathrm{Q} 221$, and run at gradients of 1.66 $\mathrm{KG} / \mathrm{in}$ and $-1.11 \mathrm{KG} / \mathrm{in}$, respectively. All new quadrupoles are 5 feet in length. 


\section{Beam Profiles and Magnet Gradients}

The $800 \mathrm{GeV}$ beam optics are compared to the $150 \mathrm{GeV}$ beam optics in Figures 4.1-4.3, for Proton, Neutrino and Meson areas, respectively. Shown in each figure are the horizontal comparisons (top half of figure) and the vertical comparisons (bottom half of figure), as a function of distance along the beam line, starting at extraction and continuing up to a representative target in each of the experimental areas. PCenter, NCenter and MWest beam lines were used. Data from the beamline SWICs are displayed as points, indicating the full width at half maximum beam sizes observed at $800 \mathrm{GeV}$. The $150 \mathrm{GeV}$ beam envelopes are drawn from TRANSPORT output, which gives half widths at the base. In general, the horizontal beam sizes agree quite well between the two cases. The $150 \mathrm{GeV}$ vertical beam extent is in general about a factor of two bigger throughout Switchyard, and approaches the $800 \mathrm{GeV}$ beam size near the production targets. 
A list of $150 \mathrm{GeV}$ quadrupole specifications is given in the following table:

$150 \mathrm{GeV}$ Quadrupole Specifications

\begin{tabular}{|c|c|c|c|}
\hline $\begin{array}{l}\text { Magnet } \\
\text { Name }\end{array}$ & $\begin{array}{c}\text { Location } \\
\text { (ft) }\end{array}$ & $\begin{array}{c}\text { Gradient } \\
\text { (KG/in) }\end{array}$ & $\begin{array}{r}\text { Current } \\
\text { (amps) }\end{array}$ \\
\hline QSK * & 86.7871 & -1.68974 & 32.36 \\
\hline Q90 & 140.9562 & 0.0 & 0.0 \\
\hline Q300 & 561.2251 & 0.72191 & 13.82 \\
\hline Q301 & 751.6579 & -0.79085 & 15.14 \\
\hline Q302 & 971.9430 & 0.90726 & 17.37 \\
\hline \multirow[t]{2}{*}{ Q303 } & 1143.1041 & 0.15665 & 3.00 \\
\hline & 1154.0006 & & \\
\hline Q304 & 1197.9085 & -0.93457 & 17.90 \\
\hline Q305 & 1383.9133 & 0.82030 & 15.71 \\
\hline Q306 & 1451.5555 & -0.54195 & 10.38 \\
\hline Q307* & 1810.9813 & 0.87009 & 16.66 \\
\hline Q308* & 1950.7298 & -1.22063 & 23.38 \\
\hline PC1Q1 & 3295.9633 & -0.59612 & 11.42 \\
\hline PC1Q2 & 3376.4281 & 0.51143 & 9.79 \\
\hline \multirow[t]{2}{*}{ PC2Q1 } & 5229.7230 & -0.40780 & 7.81 \\
\hline & 5241.0560 & & \\
\hline \multirow[t]{3}{*}{ PC3Q1 } & 5358.1420 & 0.47041 & 9.01 \\
\hline & 5369.4750 & & \\
\hline & 5380.8080 & & \\
\hline \multirow[t]{2}{*}{ Q100 } & 572.2158 & 0.58893 & 11.28 \\
\hline & 580.7158 & & \\
\hline Q101 & 651.9885 & -0.84337 & 16.15 \\
\hline Q106* & 1260.2944 & -0.37852 & 7.25 \\
\hline \multirow[t]{2}{*}{ Q120 } & 2165.8806 & -0.42467 & 8.13 \\
\hline & 2176.8801 & & \\
\hline \multirow[t]{2}{*}{ Q121 } & 2212.7756 & 0.36816 & 7.05 \\
\hline & 2223.7750 & & \\
\hline
\end{tabular}




\begin{tabular}{|c|c|c|c|}
\hline \multirow[t]{2}{*}{ NC1Q1 } & 2861.9881 & \multirow{2}{*}{0.55015} & \multirow[t]{2}{*}{10.54} \\
\hline & 2873.1537 & & \\
\hline NC1Q2A & 2950.0447 & -0.62605 & 11.99 \\
\hline NC1Q2B & 2961.0653 & -0.62605 & 11.99 \\
\hline \multirow[t]{2}{*}{ NC1Q3 } & 2972.1068 & -0.76161 & 14.58 \\
\hline & 2983.2108 & & \\
\hline Q201 & 1389.9755 & -0.77278 & 14.80 \\
\hline \multirow[t]{2}{*}{ Q202 } & 1525.3526 & 0.63834 & 12.22 \\
\hline & 1547.2733 & & \\
\hline Q203 & 1670.5358 & -0.57993 & 11.11 \\
\hline \multirow[t]{2}{*}{ Q204 } & 2044.1964 & 0.44468 & 8.52 \\
\hline & 2055.0468 & & \\
\hline \multirow[t]{2}{*}{ Q205 } & 2103.7453 & -0.58656 & 11.23 \\
\hline & 2114.7320 & & \\
\hline Q210 & 2336.1336 & 0.41844 & 8.01 \\
\hline $\mathrm{Q} 220 *$ & 2944.2253 & -1.66557 & 31.90 \\
\hline Q221 * & 2969.4000 & 1.10587 & 21.18 \\
\hline MW2Q1 & 4524.7225 & 0.75932 & 14.54 \\
\hline MW2Q2 & 4588.6816 & -0.79176 & 15.16 \\
\hline \multirow[t]{3}{*}{ MW5Q1 } & 5506.0181 & 0.70375 & 13.48 \\
\hline & 5516.8254 & & \\
\hline & 5527.6326 & & \\
\hline \multirow[t]{4}{*}{ MW5Q2 } & 5548.2645 & -0.70907 & 13.58 \\
\hline & 5559.0718 & & \\
\hline & 5569.8790 & & \\
\hline & 5580.6862 & & \\
\hline
\end{tabular}

The asterisk $\left({ }^{*}\right)$ indicates the new 5 -foot quadrupoles. 


\section{PROTON}

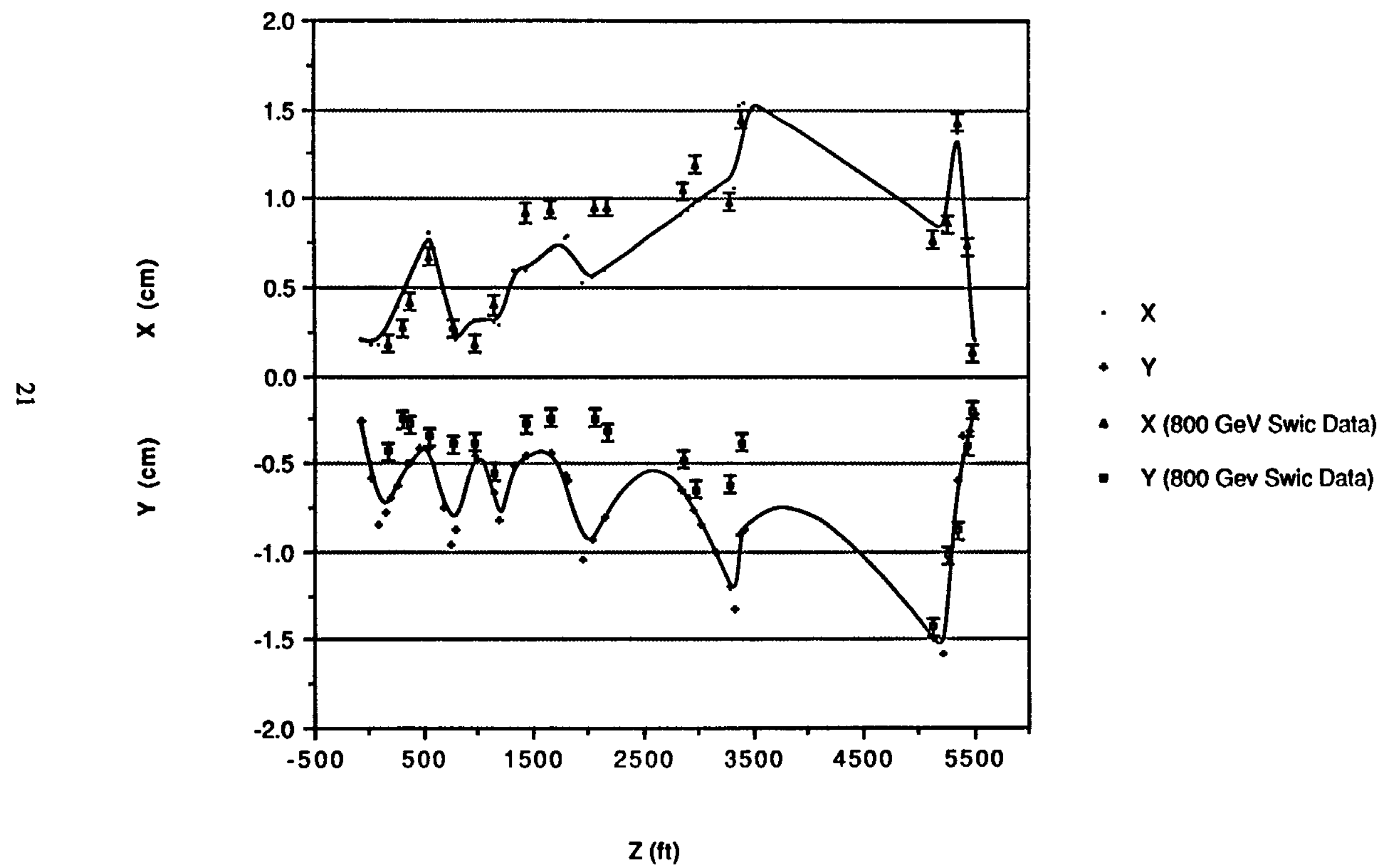

Figure 4.1 
NEUTRINO

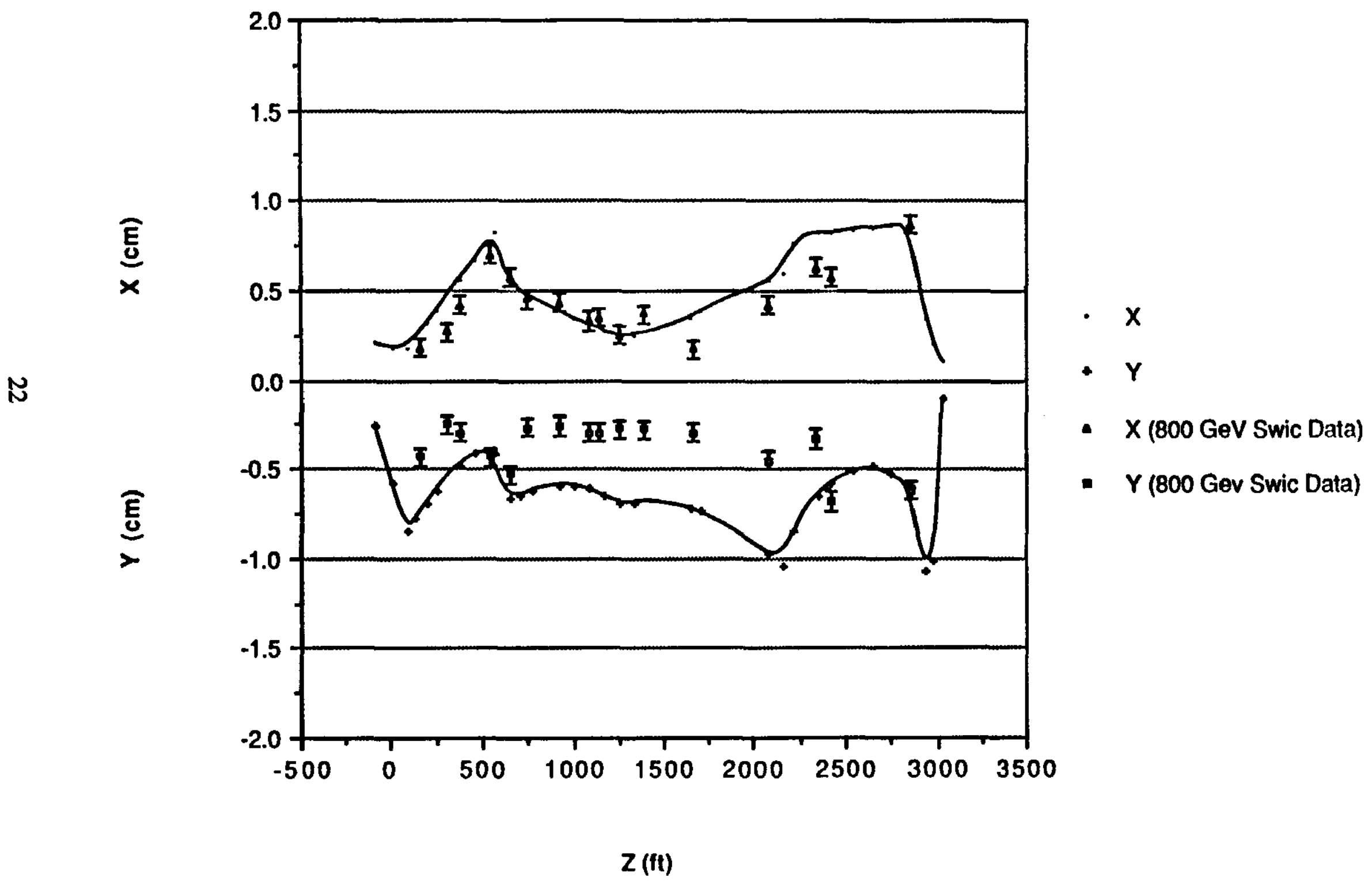

Figure 4.2 


\section{MESON}

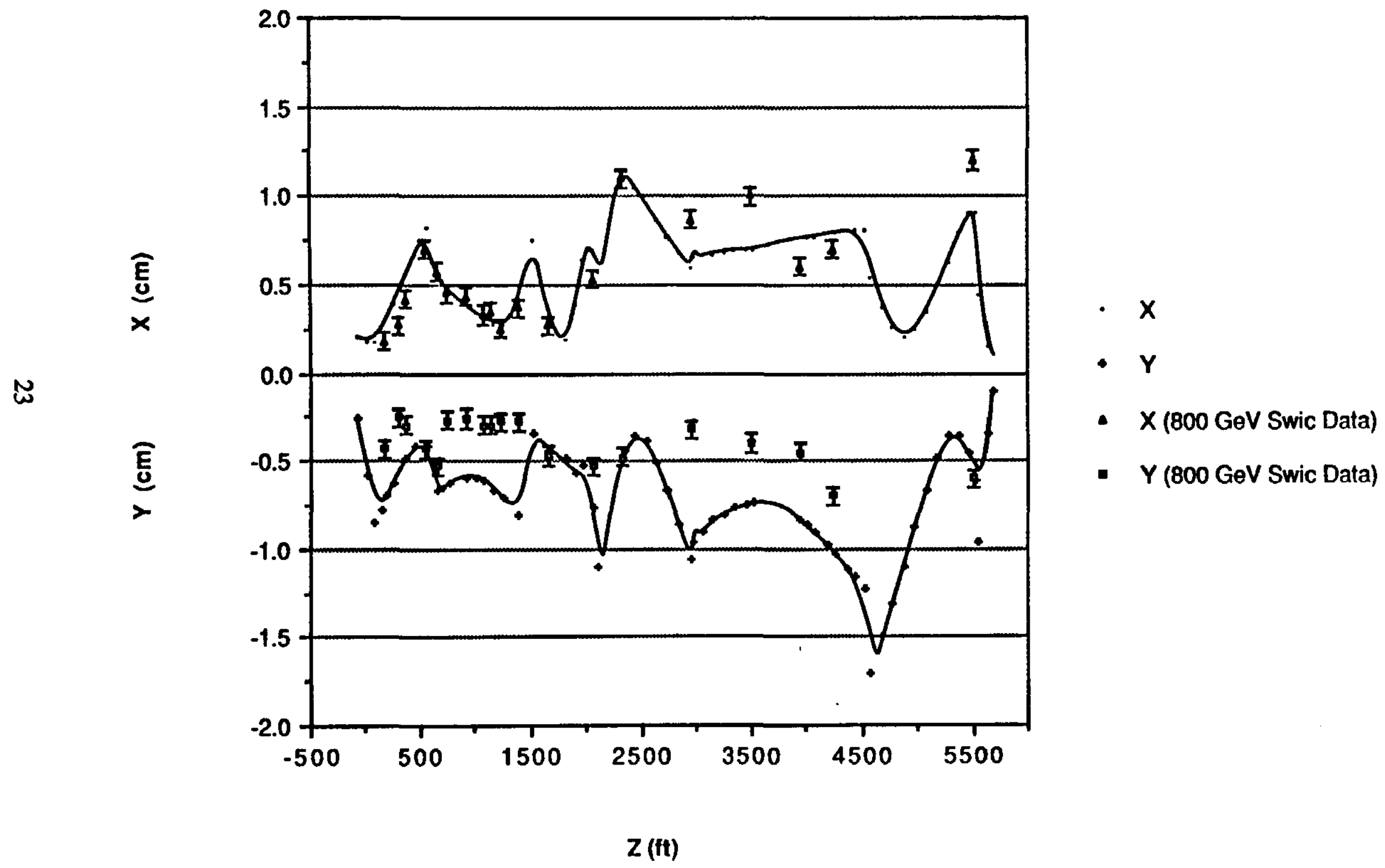

Figure 4.3 


\section{Quadrupole Power Supply Regulation}

Many of the Switchyard quadrupole currents for $150 \mathrm{GeV}$ are less than 10 amps. Trying to use a 100 amp power supply at these low values has historically proven difficult. For our purpose, the question is: How can good regulation be attained at both $150 \mathrm{GeV}$ and $800 \mathrm{GeV}$ ? The Research Division has conducted studies on this question in the past; when new 100 amp PEI supplies were purchased, specifications were worked out between the Electrical Department and the vendor to try to achieve good regulation from 1 to 100 amps, without enormous costs. According to A. Visser, quality temperature sensitive transductors were purchased from a Swiss manufacturer, highly accurate burden resistors were purchased (a few parts per million), and the electronic control cards were rebuilt. Past effort focused only on the last of these, with the electronics attempting to compensate for everything. Looking at a wider picture has proven to be much more successful.

Based on this experience, all the ACME power supplies used in the Research Division beam lines were upgraded during the summer of 1988 at a cost of about $\$ 1000 /$ unit. A. Visser felt confident that just by using these modified supplies, or ones having similar modifications ( such as Switchyard HP 100/100's ), one could simultaneously attain $100 . \pm 0.1 \%$ amps at the top end and something like $1 . \pm 0.2 \%$ amps at the low end. This should fit our requirements.

A. Visser also pointed out that at these levels, the absolute value of the field depends not only on the power supply, but on the hysteresis of the magnet. A magnet that has had its polarity reversed will take a lot less current out of the power supply than one that has not been reversed. In order to account for this, it would be useful to map the field integral of the $3 Q 120$ in the region of 1 . to 10 . amps. 


\subsection{New High Intensity Line to Neutrino Area}

Design criteria for the new Switchyard high intensity primary beam line from the Main Injector to the Neutrino area were that the following conditions be satisfied, to minimize costs:

The new line should stay within existing Switchyard tunnels, with a minimum of civil construction.

Trajectory through the Switchyard beam dump should remain within the existing beam transmission pipe, thus avoiding major civil construction involving the beam dump.

A design has been found which satisfies both of these criteria, with no major compromise required.

The extracted Main Injector beam in the dedicated High Intensity line is initially to the West of the PSEP splitting station. As shown in Figure $4.4 \mathrm{a}$ the beam must be transferred to the East side of the existing Neutrino line Switchyard elements through Enc. B. These elements are already mounted at the West wall of this enclosure. This beam shift is accomplished with two EPB dipoles beside the PSEPs. The beam is then brought back roughly parallel to the existing Neutrino line with two horizontal West bending EPB dipoles and one vertical EPB. Cross sections of different beam line elements through Enc. B are shown in Figure 4.4b.

Civil construction is required for new berm pipe between Enc. B and Enc. C. In general, new pipe is required between each enclosure; as the existing eight inch diameter pipes are completely shadowed by present Switchyard magnets, with no room for a new beam. This is typically less expensive by an order of magnitude than adding new tunnel sections, however. 
In Enc. $C$, the new beam line remains to the East of the Tevatron Neutrino line, as shown in Figure 4.5a. Beam element cross sections in Enc. $\mathrm{C}$ are given in Figure $4.5 \mathrm{~b}$. The major hurdle at this point becomes compatibility with the Switchyard dump, located between Enc. C and G1.

The dump has an eight inch diameter transmission pipe through the $26 \mathrm{ft}$. Iong core box. Figure 4.7 shows this core box during initial Switchyard construction. The entrance hole to the dump itself is near the dump box center, while the transmission hole is toward the top.

The new beam trajectory is designed to cross the existing Tevatron beam at a point near the beam dump, thus sharing the same transmission beam pipe. New berm pipe is required both upstream and downstream of this region, but civil construction at the beam dump is not required.

In Enc. G1, the new beam thus is to the West of the existing line. This is required by tunnel constraints, as shown in Figure 4.6a. and 4.6b. Space is very marginal in $\mathrm{G} 1$ for a dipole to orient the beam trajectory toward G2, as shown in Figure 4.6a. Spatial constraints may require a short tunnel extension at G1, the only location where new tunnel length is being considered. An extension at this point also aids Tevatron Switchyard transport for energies above $800 \mathrm{GeV}$, enabling the addition of another Lambertson magnet. Total desired new tunnel length at $\mathrm{G} 1$ would be about $35 \mathrm{ft}$.

New berm pipe is again required between Enc $G 1$ and $G 2$ for most of this distance. A large existing pipe, due to Muon line requirements, enables the new beam to utilize a portion of this pipe. In G2 the new beam is transmitted above the Muon line as shown in Figure $4.6 \mathrm{a}$ and $4.6 \mathrm{~b}$. The beam then intersects with the Tevatron Neutrino line at a point upstream of the NLAM string. From this point the beam shares a common trajectory with the existing $\mathrm{NC}$ beam to NeuHall. 
Total civil construction requirements have been kept to a minimum. Besides the short $35 \mathrm{Ft}$. tunnel addition in $\mathrm{G1}$, a total of about $670 \mathrm{Ft}$. of new berm pipe will be required between existing Switchyard tunnels.

Beam optics for the dedicated High Intensity line through the Switchyard are shown in Figure 4.8. As narrow aperture electrostatic septa and Lambertsons are not utilized in this beam line, the beam optics envelope is very well constrained.

Required magnet elements for the new $120-150 \mathrm{GeV}$ line are given in the following table. The table gives the coordinates of the bend points for the EPB dipoles, Quads and Trim dipoles used in the dedicated High Intensity beam transport design. Additionally, magnetic field requirements and power supply loads are shown. 
Neutrino High Intensity $150 \mathrm{GeV}$ Beamline

\begin{tabular}{|c|c|c|c|c|c|c|c|c|}
\hline nclosure & Name & $\begin{array}{l}\text { Magnet Type } \\
N=\text { New } \\
\text { E=Existing }\end{array}$ & & $\begin{array}{c}\text { Station } \\
(F t)\end{array}$ & $\begin{array}{l}\text { Length } \\
(F t)(K G\end{array}$ & $\begin{array}{l}\text { Field } \\
\text { or } K G / i n)\end{array}$ & $\begin{array}{l}\text { Current } \\
\text { (Amps) }\end{array}$ & loltage \\
\hline $\begin{array}{l}\text { ransfer Hall } \\
\text { ransfer Hall } \\
\text { ransfer Hall }\end{array}$ & $\begin{array}{l}Q .501 \\
H .501-1 \\
H .501 .2\end{array}$ & $\begin{array}{l}\text { Quadrupole } \\
\text { EPB Dipole } \\
\text { EPB Dipole }\end{array}$ & $\begin{array}{l}N \\
N \\
N\end{array}$ & $\begin{array}{r}51.5 \\
214.5 \\
225.5\end{array}$ & $\begin{array}{l}5 \\
10 \\
10\end{array}$ & $\begin{array}{l}-2.140 \\
10.707 \\
10.707\end{array}$ & $\begin{array}{r}41.0 \\
1038.6 \\
1038.6\end{array}$ & $\begin{array}{l}46.1 \\
18.2 \\
18.2\end{array}$ \\
\hline $\begin{array}{l}\text { ncl. B } \\
\text { ncl. B } \\
\text { ncl. B } \\
\text { ncl. B } \\
\text { ncl. B } \\
\text { ncl. B } \\
\text { nd. B }\end{array}$ & $\begin{array}{l}H .502-1 \\
H .502-2 \\
V-503 \\
Q-502 \\
Q-503 \\
H T .504 \\
\text { VT.505 }\end{array}$ & $\begin{array}{l}\text { EPB Dipole } \\
\text { EPB Dipole } \\
\text { EPB Dipole } \\
\text { Quadrupole } \\
\text { Quadrupole } \\
\text { Trim } \\
\text { Trim }\end{array}$ & $\begin{array}{l}N \\
N \\
N \\
N \\
N \\
N \\
N\end{array}$ & $\begin{array}{l}426.5 \\
437.5 \\
448.5 \\
771.5 \\
786.5 \\
791.5 \\
795.5\end{array}$ & $\begin{array}{l}10 \\
10 \\
10 \\
5 \\
5 \\
3 \\
3\end{array}$ & $\begin{array}{r}11.015 \\
11.015 \\
6.023 \\
3.083 \\
-3.173\end{array}$ & $\begin{array}{r}1068.5 \\
1068.5 \\
584.2 \\
59.0 \\
60.8\end{array}$ & $\begin{array}{l}18.7 \\
18.7 \\
10.2 \\
66.4 \\
68.4\end{array}$ \\
\hline $\begin{array}{l}\text { nol. C } \\
\text { nol. C } \\
\text { ncl. C } \\
\text { ncl. C }\end{array}$ & $\begin{array}{l}\text { VH-506-1 } \\
\text { VH-506-2 } \\
\text { HT }-507 \\
\text { VT }-508\end{array}$ & $\begin{array}{l}\text { EPB Dipole } \\
\text { EPB Dipole } \\
\text { Trim } \\
\text { Trim }\end{array}$ & $\begin{array}{l}N \\
N \\
N \\
N\end{array}$ & $\begin{array}{l}1159.5 \\
1170.5 \\
1178.0 \\
1182.0\end{array}$ & $\begin{array}{c}10 \\
10 \\
3 \\
3\end{array}$ & $\begin{array}{l}97 \\
97\end{array}$ & & $\begin{array}{l}17.3 \\
17.3\end{array}$ \\
\hline $\begin{array}{l}\text { nd. G1 } \\
\text { nd. G1 } \\
\text { nd. G1 }\end{array}$ & $\begin{array}{l}H .510 \\
H T-511 \\
V T-512\end{array}$ & $\begin{array}{l}\text { EPB Dipole } \\
\text { Trim } \\
\text { Trim }\end{array}$ & $\begin{array}{l}N \\
N \\
N\end{array}$ & $\begin{array}{l}1711.5 \\
1725.0 \\
1729.0\end{array}$ & $\begin{array}{l}10 \\
3 \\
3\end{array}$ & 4.773 & .0 & 8.1 \\
\hline $\begin{array}{l}\text { End. G2 } \\
\text { End. G2 } \\
\text { End. G2 } \\
\text { End. G2 } \\
\text { Endl. G2 } \\
\text { Endl. G2 } \\
\text { End. G2 } \\
\text { End. G2 } \\
\text { End. G2 } \\
\text { End. G2 }\end{array}$ & $\begin{array}{l}V-520-1 \\
V-520-2 \\
Q-520 \\
H-521 \\
Q .521 \\
V-522 \\
H .523 \\
\text { NOOH } \\
\text { NOOV } \\
\text { NLAM-1/4 }\end{array}$ & $\begin{array}{l}\text { EPB Dipole } \\
\text { EPB Dipole } \\
\text { Quadrupole } \\
\text { EPB Dipole } \\
\text { Quadrupole } \\
\text { EPB Dipole } \\
\text { EPB Dipole } \\
\text { Trim } \\
\text { Trim } \\
\text { Lambertsons }\end{array}$ & $\begin{array}{l}N \\
N \\
N \\
N \\
N \\
N \\
N \\
E \\
E \\
E\end{array}$ & $\begin{array}{l}2099.5 \\
2110.5 \\
2119.0 \\
2140.0 \\
2158.5 \\
2304.0 \\
2315.0 \\
2344.0 \\
2347.0 \\
2373.3\end{array}$ & $\begin{array}{l}10 \\
10 \\
5 \\
10 \\
5 \\
10 \\
10 \\
3 \\
3 \\
10\end{array}$ & $\begin{array}{r}7.692 \\
7.692 \\
1.647 \\
13.678 \\
-1.907 \\
12.079 \\
13.075\end{array}$ & $\begin{array}{r}746.1 \\
746.1 \\
31.5 \\
1326.8 \\
36.5 \\
1171.7 \\
1268.3\end{array}$ & $\begin{array}{l}13.0 \\
13.0 \\
35.4 \\
23.2 \\
41.1 \\
20.5 \\
22.2\end{array}$ \\
\hline $\begin{array}{l}\text { ncl. N01 } \\
\text { ncl. N01 } \\
\text { ncl. N01 } \\
\text { ncl. N01 } \\
\text { ncl. N01 } \\
\text { ncl. N01 }\end{array}$ & $\begin{array}{l}\text { N01Q1-1 } \\
\text { N01Q1-2 } \\
\text { N01DE-1/5 } \\
\text { N01Q2-1 } \\
\text { N01Q2-2 } \\
\text { N01DE-5/9 }\end{array}$ & $\begin{array}{l}\text { Quadrupole } \\
\text { Quadrupole } \\
\text { EPB Dipoles } \\
\text { Quadrupole } \\
\text { Quadrupole } \\
\text { EPB Dipoles }\end{array}$ & $\begin{array}{l}E \\
E \\
E \\
E \\
E \\
E\end{array}$ & $\begin{array}{l}2862.0 \\
2873.0 \\
2917.0 \\
2950.0 \\
2961.0 \\
2917.0\end{array}$ & $\begin{array}{l}10 \\
10 \\
10 \\
10 \\
10 \\
10\end{array}$ & $\begin{array}{r}.474 \\
.474 \\
2.533 \\
-1.179 \\
-1.179 \\
2.533\end{array}$ & $\begin{array}{r}9.1 \\
9.1 \\
245.7 \\
22.6 \\
22.6 \\
245.7\end{array}$ & $\begin{array}{l}20.5 \\
20.5 \\
21.5 \\
50.9 \\
50.9 \\
17.2\end{array}$ \\
\hline
\end{tabular}

Totals

5 New Quadrupoles

13 New EPB Dipoles

6 New Trims 


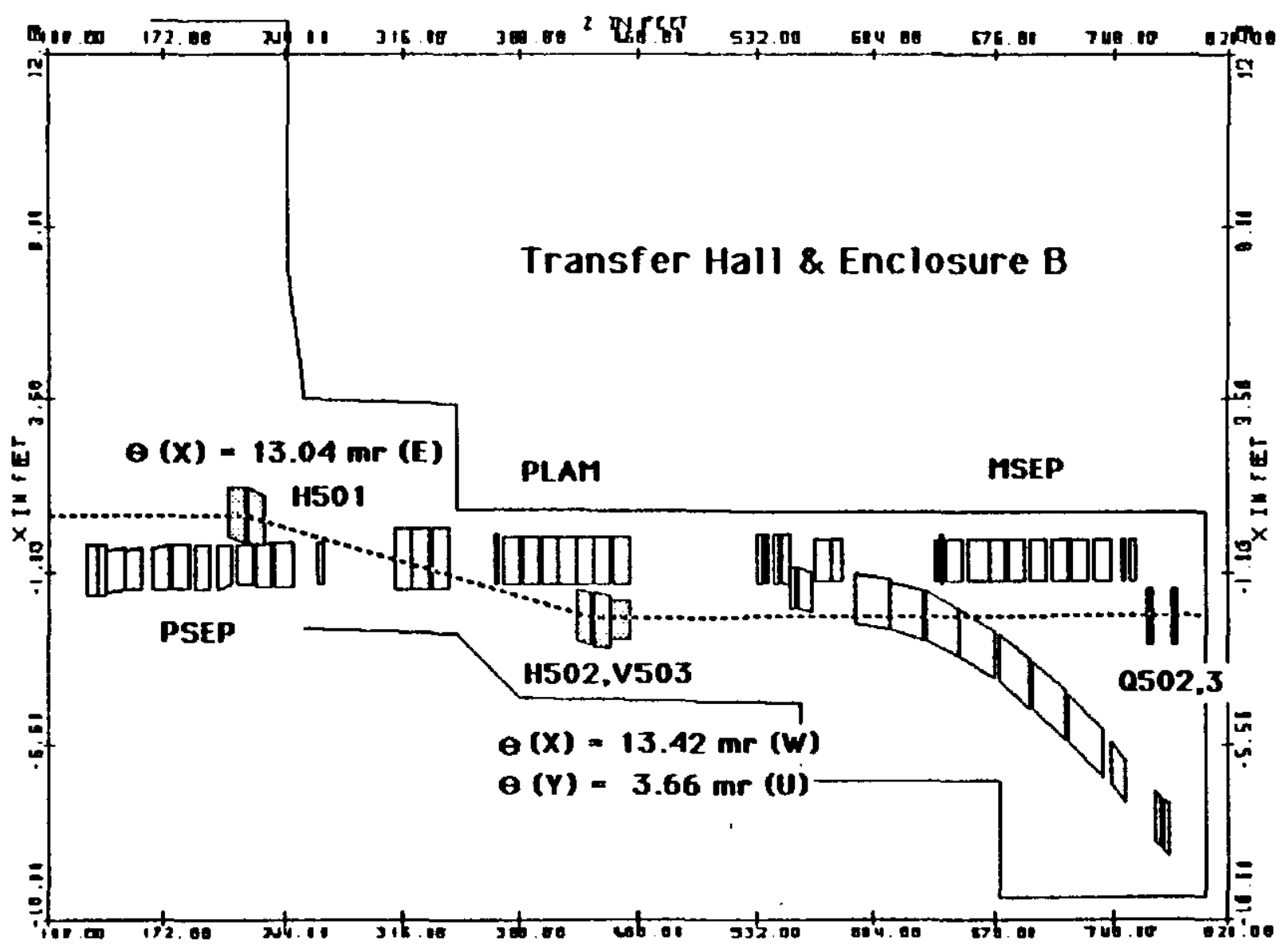

Figure $4.4 a$ 
VH94-1-US Tunnel Cross Sections - Encl. B

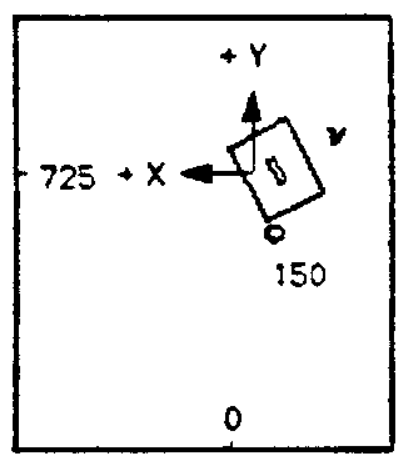

$\begin{array}{lll}v & z=311.17 \mathrm{ft} \\ 150 \mathrm{GeV} & x=-.70 \mathrm{ft} & y=725.08 \mathrm{ft} \\ \text { WWall/floor } & x=-.69 \mathrm{ft} & y=724.06 \mathrm{ft} \\ \text { EWall/Ceiling } & x=-2.42 \mathrm{ft} & y=720.56 \mathrm{ft} \\ & x=-58 \mathrm{ft} & y=727.56 \mathrm{ft}\end{array}$
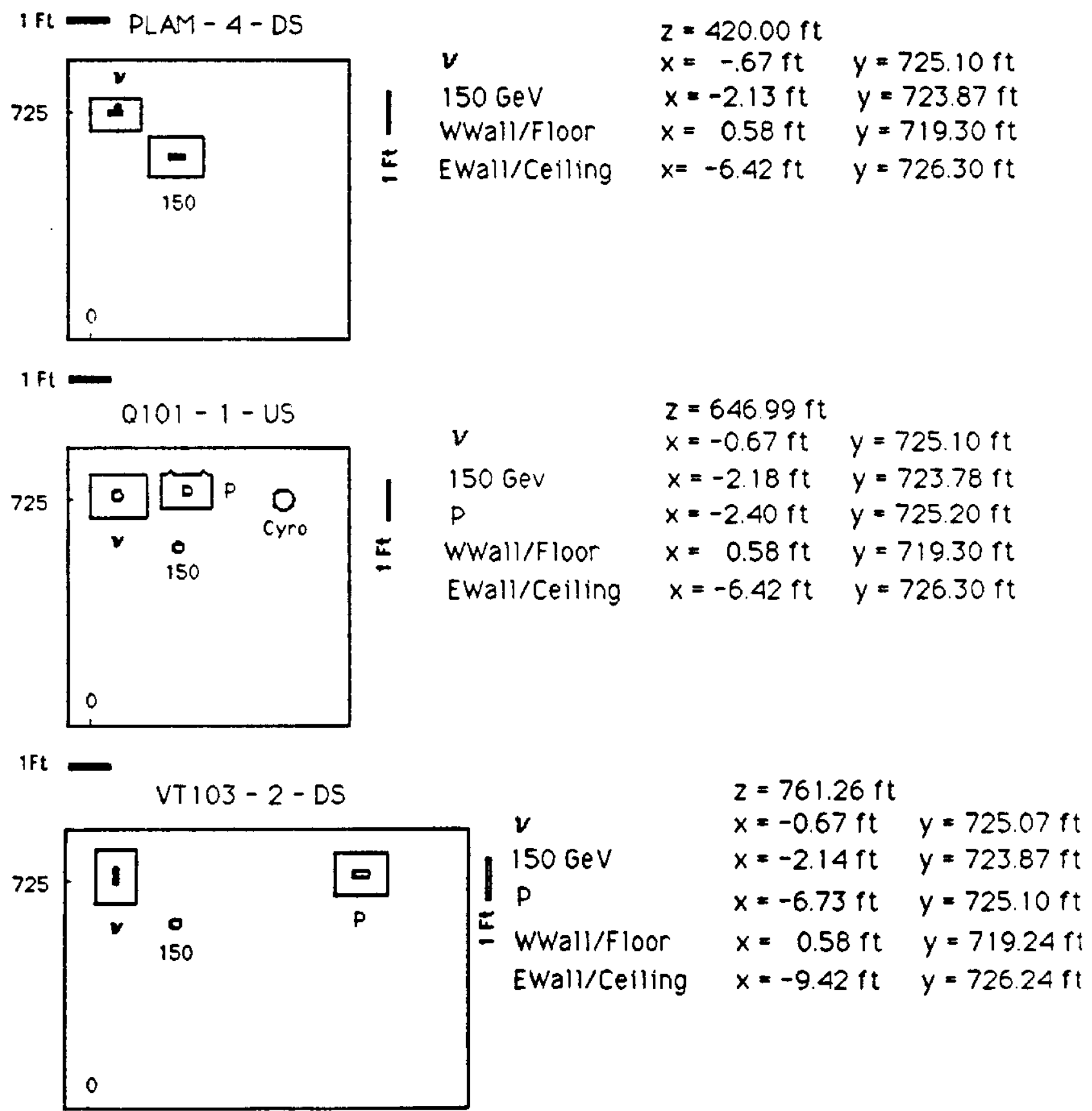


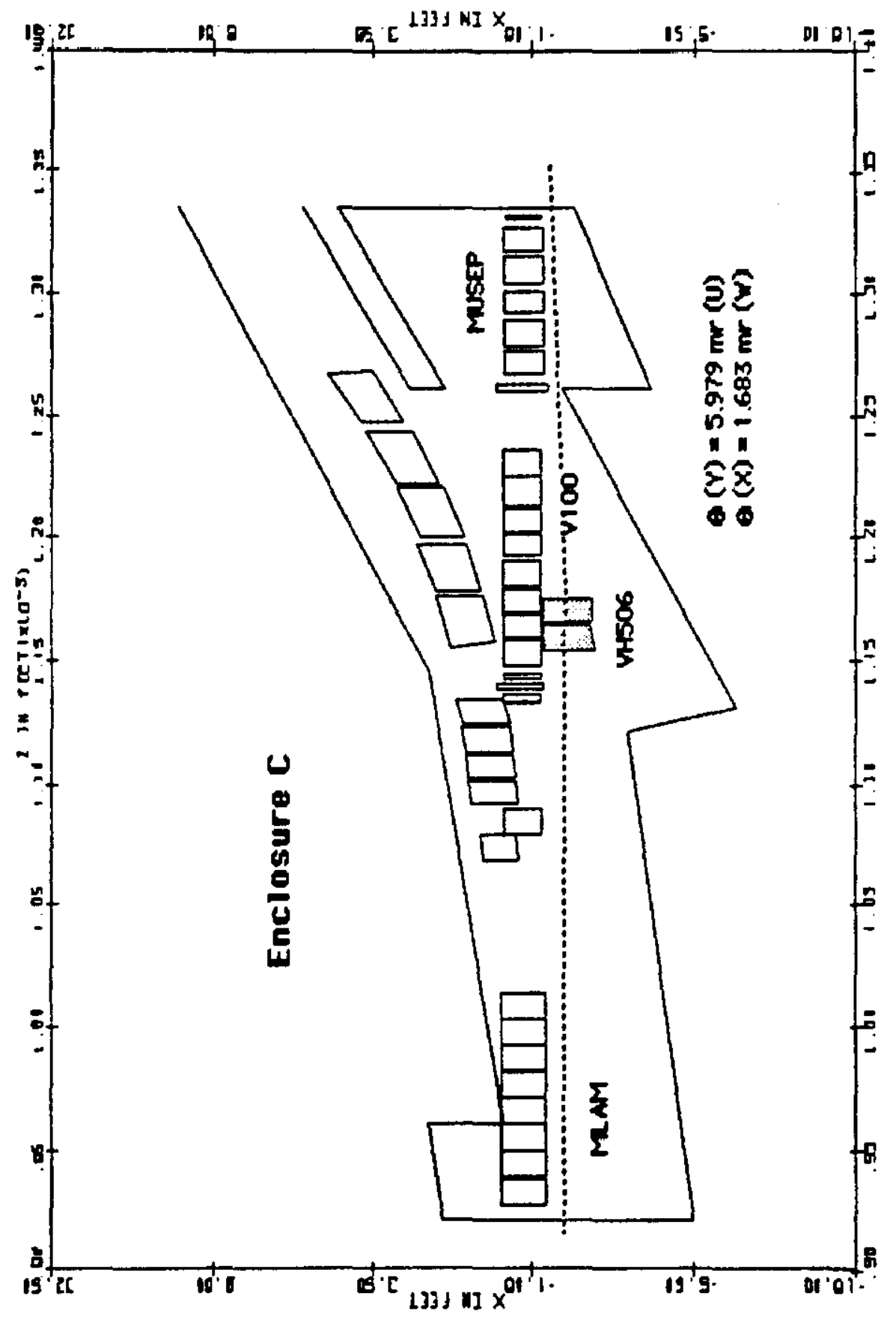

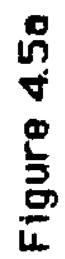


Tunnel Cross Sections - Encl. C

MLAM - 8 - DS

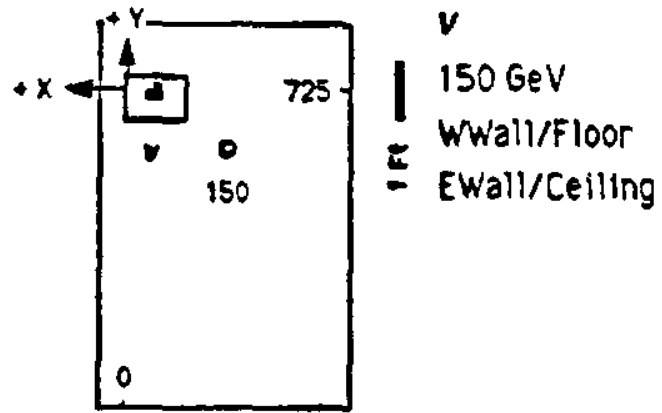

$z=1014.64 \mathrm{ft}$

$x=-0.67 \mathrm{ft} \quad y=725.05 \mathrm{ft}$

$x=-2.05 \mathrm{ft} \quad y=723.94 \mathrm{ft}$

$x=0.48 \mathrm{ft} \quad y=719.22 \mathrm{ft}$

$x=-4.52 \mathrm{ft}$

$y=725.22 \mathrm{ft}$
$1 F t=-D S$
$V 100-7-D S$

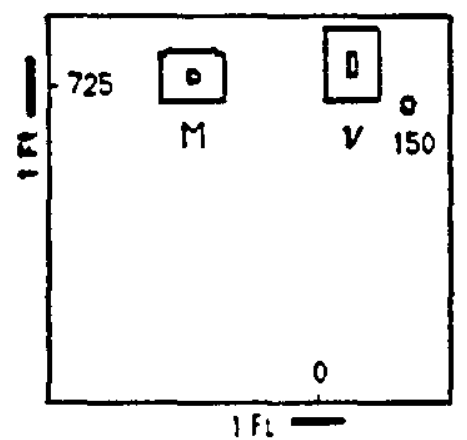

\begin{tabular}{c}
$0105-U 5$ \\
\hline
\end{tabular}

End EnCl C - SY Dump

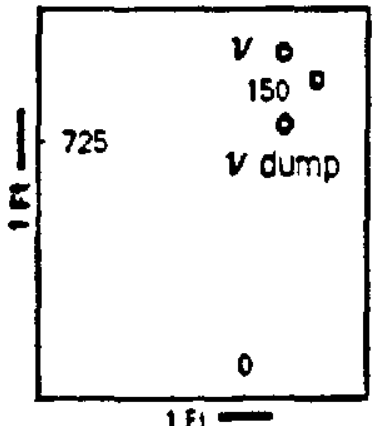

$z=1223.98 \mathrm{ft}$

$v \quad x=-0.69 \mathrm{ft} \quad y=725.41 \mathrm{ft}$

$M \quad x=2.49 \mathrm{ft} \quad y=725.20 \mathrm{ft}$

$150 \mathrm{GeV} \quad x=-1.77 \mathrm{ft} \quad y=724.70 \mathrm{ft}$.

W'Wall/Floor $\quad x=5.35 \mathrm{ft} \quad y=719.33 \mathrm{ft}$

EWall/Ceiling $x=-2.65 \mathrm{ft} \quad y=726.33 \mathrm{ft}$ $z=1259.91 \mathrm{ft}$

$x=-0.70 \mathrm{ft} \quad y=725.76 \mathrm{ft}$

$150 \mathrm{GeV} \quad x=-1.64 \mathrm{ft} \quad y=725.14 \mathrm{ft}$

Wwall/floor $\quad x=1.50 \mathrm{ft} \quad y=719.22 \mathrm{ft}$

EWall/Celing $x=-1.66 \mathrm{ft} \quad y=726.22 \mathrm{ft}$

Figure $4.5 b$ 


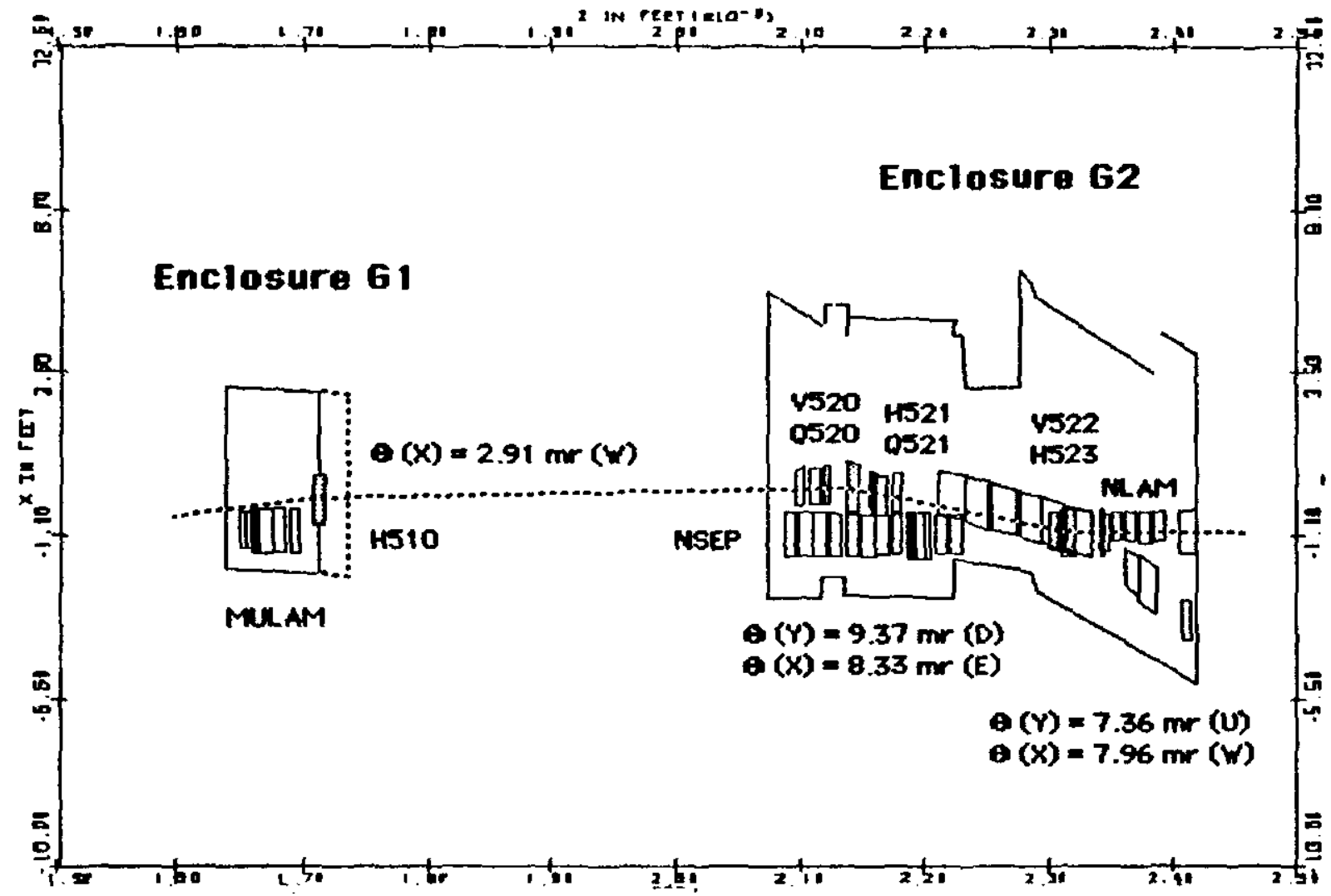

Figure 4.60 
Tunnel Cross Sections - Encl. G1 \& G2

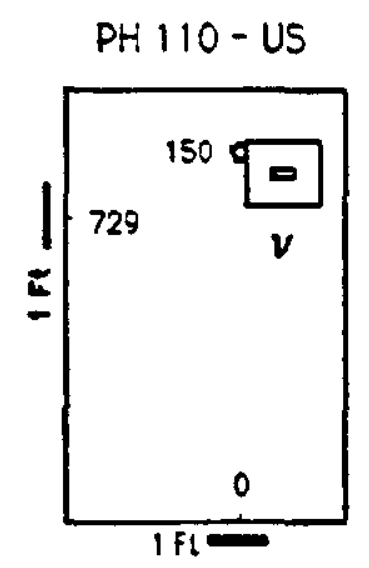

$$
\begin{array}{ll}
z=1657.65 \mathrm{ft} & \\
x=-0.81 \mathrm{ft} & y=729.66 \mathrm{ft} \\
x=-0.05 \mathrm{ft} & y=730.02 \mathrm{ft} \\
x=3.07 \mathrm{ft} & y=731.01 \mathrm{ft} \\
x=-1.94 \mathrm{ft} & y=724.01 \mathrm{ft}
\end{array}
$$

$150 \mathrm{GeV} \quad \mathrm{EWall} / \mathrm{Ce}$ lling $\quad x=3.07 \mathrm{ft} \quad y=731.01 \mathrm{ft}$ Wwall/floor $\quad x=-1.94 \mathrm{ft}$

GI

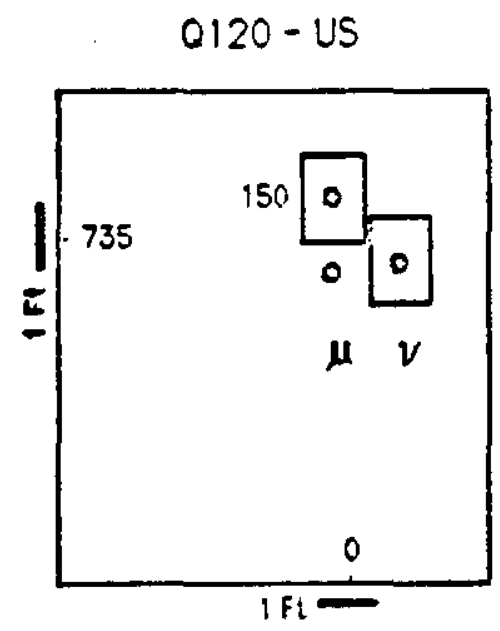

$z=2160.55 \mathrm{ft}$

$x=-0.95 \mathrm{ft}$

$y=734.59 \mathrm{ft}$

$v$

$150 \mathrm{GeV}$

$x=0.26 \mathrm{ft}$

$y=735.63 \mathrm{ft}$

$\mu$

$x=0.26 \mathrm{ft}$

$y=734.41 \mathrm{ft}$

EWall/Ceiling

$x=5.09 \mathrm{ft}$

$y=737.38 \mathrm{ft}$

W'all/Floor

$x=-2.51 \mathrm{ft}$

$y=729.38 \mathrm{ft}$

G2

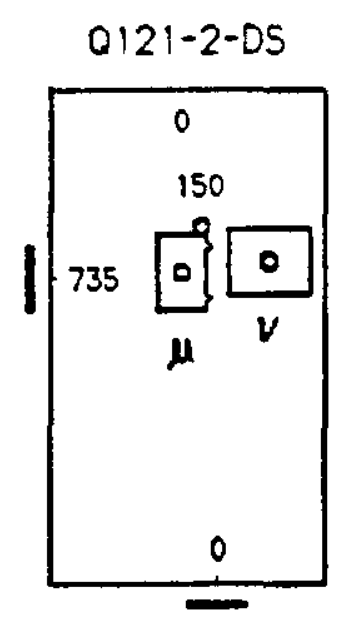

$z=2228.93 \mathrm{ft}$

$x=-0.94 \mathrm{ft}$

$y=735.27 \mathrm{ft}$

$x=0.26 \mathrm{ft}$

$y=735.83 \mathrm{ft}$

$150 \mathrm{GeV}$

$\mu$

$x=0.62 \mathrm{ft}$

$y=735.10 \mathrm{ft}$

EWall/Ceiling

$x=2.96 \mathrm{ft}$

$y=738.06 \mathrm{ft}$

WWall/Floor

$x=-1.94 \mathrm{ft}$

$y=730.06 \mathrm{ft}$

G2

Figure $4.6 b$ 


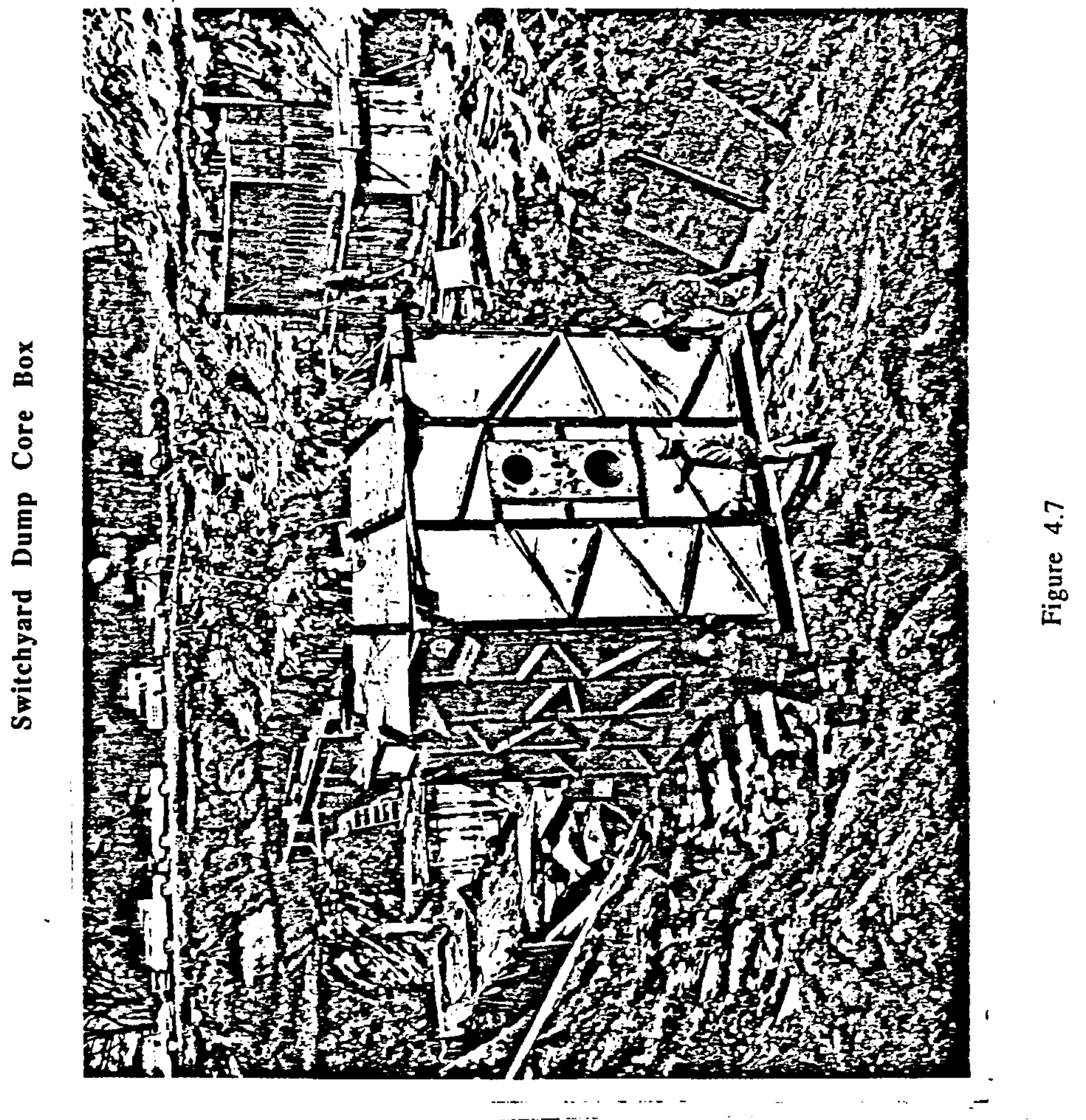


Neutrino High Intensity $150 \mathrm{GeV}$ Beamline

FWHM $(X, Y)=2.35$ Sigma $=(X, Y)=98 \%$ Beam

a

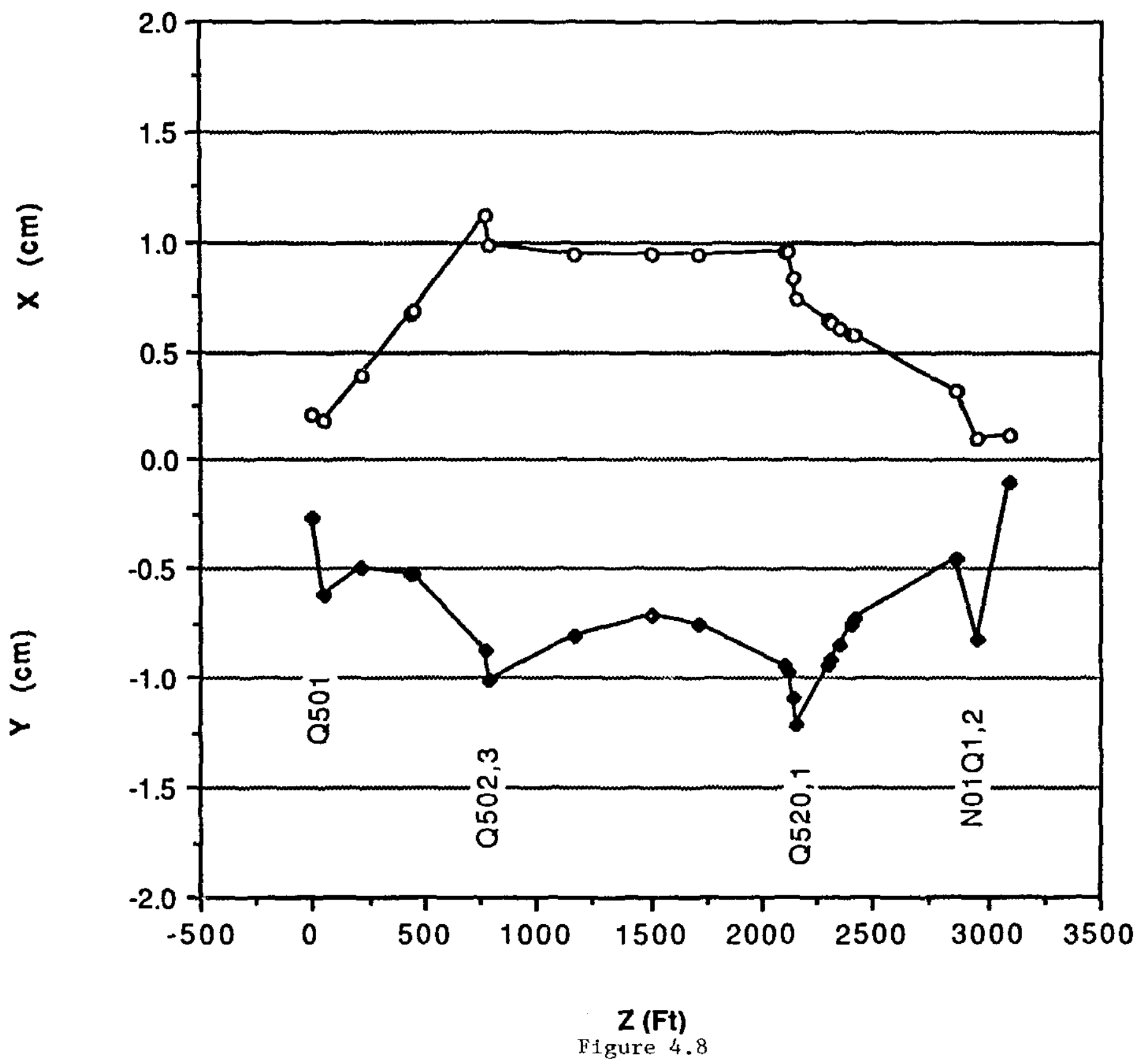




\section{Experimental Facilities at $120-150 \mathrm{GeV}$}

\subsection{The Development of a Model for High Intensity Facilities}

In order to evaluate the feasibility and cost of a High Intensity program at $120-150 \mathrm{GeV}$, a model was developed based on several assumptions. It was assumed, for example, that low intensity beams of this energy range would be made available to all three experimental areas for use as test beams and to calibrate and debug experiments. This beam would be made available during collider runs. Because an extraction system to the existing experimental areas would be in place, it followed that the high intensity beam could use this same extraction system, rather than require an additional extraction system to a new experimental area.

It was found that a high luminosity $150 \mathrm{GeV}$ beam during Tevatron fixed target running requires a new beam transport system, even though the beam is to be transported to an existing experimental area. The alternative of time sharing with the $800 \mathrm{GeV}$ beam was discarded as unproductive for physics potential and operationally difficult. To minimize the cost of additional beam transport, it has been assumed that only one area will be refitted as a High Intensity area.

All three areas were considered for this purpose, and it was apparent that the choice of location for High Intensity experiments is dependent upon the specific program considered. For the purposes of this model, the Neutrino Area was chosen as the location for High Intensity transport, but other facilities could be developed in the Meson or Proton Areas as well. As will be explained in the next paragraph, the Neutrino Area was chosen because the model deals with neutrino and kaon experiments. The most cost effective way to do the neutrino experiments was to utilize the existing facilities in the Neutrino Area.

A model was developed for experiments that could be done only with High Intensity beams. These experiments are therefore uniquely dependent on the availability of 120-150 GeV 
beam with 20 pulses per minute and intensities up to $3 \times 10^{13}$ ppp. Two experiments were identified in this category: Rare kaon decay experiments, and neutrino oscillation experiments that involve a tau neutrino. The potential for kaon physics at this type of facility was evaluated by a committee chaired by B. Winstein; a report of the committee should be available later this year. The neutrino oscillation experiment was suggested by $H$. Harari in a series of lectures given at Fermilab in the summer of 1988. The potential of such an experiment was further advanced by $\mathrm{N}$. Reay in the Neutrino Workshop held at Fermilab in the Fall of 1988. It must be understood that these experiments have not been formally proposed by the proponents, but are considered here as potential users of this new capability contingent on successful completion of the normal Laboratory review and acceptance process. This model was developed to help evaluate the feasibility of carrying out these types of experiments at Fermilab.

The conclusion reached, from detailed consideration of such experiments, is that the possibilities for a High Intensity program exist in the Neutrino Area and could be developed on a time scale that would be interesting for the physics goals involved.

\subsection{High Sensitivity Kaon Experiment}

\section{The Kaon Experiment}

The kaon experiment would be housed in a building of approximate dimensions $100^{\prime}$ long, $40^{\prime}$ wide and 30' high. The building must have good crane coverage to support the construction of the spectrometer magnet. A schematic of the apparatus as given by Winstein et.al. (EFI preprint 89-01) is shown in figure 5.1. In addition to the experimental hall, provision would also be made for a counting room with rough dimensions of $50^{\prime}$ by $50^{\prime}$. A nearby service building would also be required to provide power and cooling water for the magnet, and a storage location for chamber gases.

Several locations for the kaon experiment were considered in the Neutrino Area with the hope of finding a location that would require minimal use of new resources. The first location considered was Neuhall, where the experiment could reside at the expense of turning off every other possible activity in the Neutrino Area. 


\section{Model neutral kaon apparatus using the Main Injector}

$\omega$

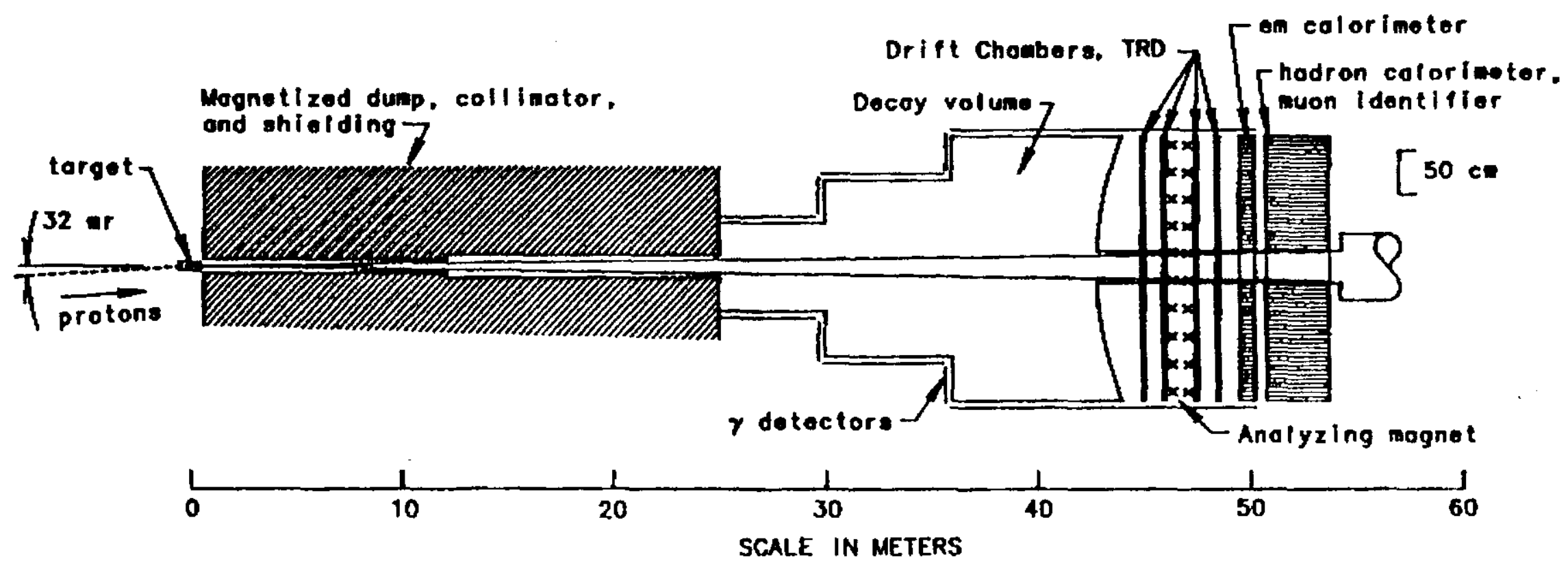

Figure 5.1 
This location failed because the sweeper and spectrometer magnets do not fit into the existing hall, which measures in places less than $15^{\prime} \mathrm{X} 15^{\prime}$ in cross-sectional area. The region east of the Neutrino berm was also considered, since a new beam line could be taken from Neuhall or enclosure NE4. This location has some potential for success, but suffers from the problem of accessibility for construction vehicles and personnel access. It was felt that the barns might have to be removed to provide access to the new building. Another location that was considered was the old Muon Lab - NWA. Conceptual design for this location proved to be very difficult because of the location of power substations, water cooling towers and a service building in the path of the berm needed for the High Intensity beam. No attempt was made to develop this concept because of these difficulties.

The location that proved to work the best was on the west side of the Neutrino berm. The beam can be brought out of Neuhall with a bend string consisting of seven EPB dipoles, as shown in Figure 5.2. Although this is a large bend string, construction of the new facility is greatly simplified outside of the berm. This location also provides convenient access to power and cooling water at the NS2 service building. Easy road access for both construction and personnel is available from Road $A$. An earth berm will be needed behind the building to eliminate muon radiation headed for Road $A$ and the Operations Center.

\section{The Kaon Beam}

The new High Intensity Kaon Facility was assumed to have its target located at $X=45, Z=4100$ ( DUSAF coordinates). The portion of this beam line upstream of the Neutrino target position, $Z=$ 3040.3, uses the same components as the existing Neutrino beam line. In the design described here, the tune used upstream of Neuhall was kept the same as the Neutrino tune. For the proposed Kaon beam the quads NC1Q1, NC1Q2A and NC1Q2B retain the same polarity but have different gradients than those used in the Neutrino tune. The NC1Q3 quads will require the opposite polarity in addition to a different gradient. 
When the Kaon beam is used, it will be necessary to remove the bed plates supporting the NC Neutrino target and any devices downstream of the target. They will be replaced (see figure 5.2) by a new 5-1.5-120 EPB dipole starting at the same $Z$ as the NC target which bends the beam West by $9.137 \mathrm{mr}$, and six new EPB dipoles located at the downstream end of the enclosure which bend West by a total of $53.38 \mathrm{mr}$. This will make the beam leave Neuhall through the downstream wall near the Northwest corner of the enclosure and will aim it at $X=45$ at $Z=4100$. A section of berm will have to be removed downstream of Neuhall to install the new beam pipe. Some of the dirt removed to install the new pipe may be radioactive since it is near the old Neutrino Target Tube. However, the problem with radioactive dirt should be tractable; previously, the installation of the NE beam pipe on the other side of the Neutrino Target Tube was accomplished without major difficulties.

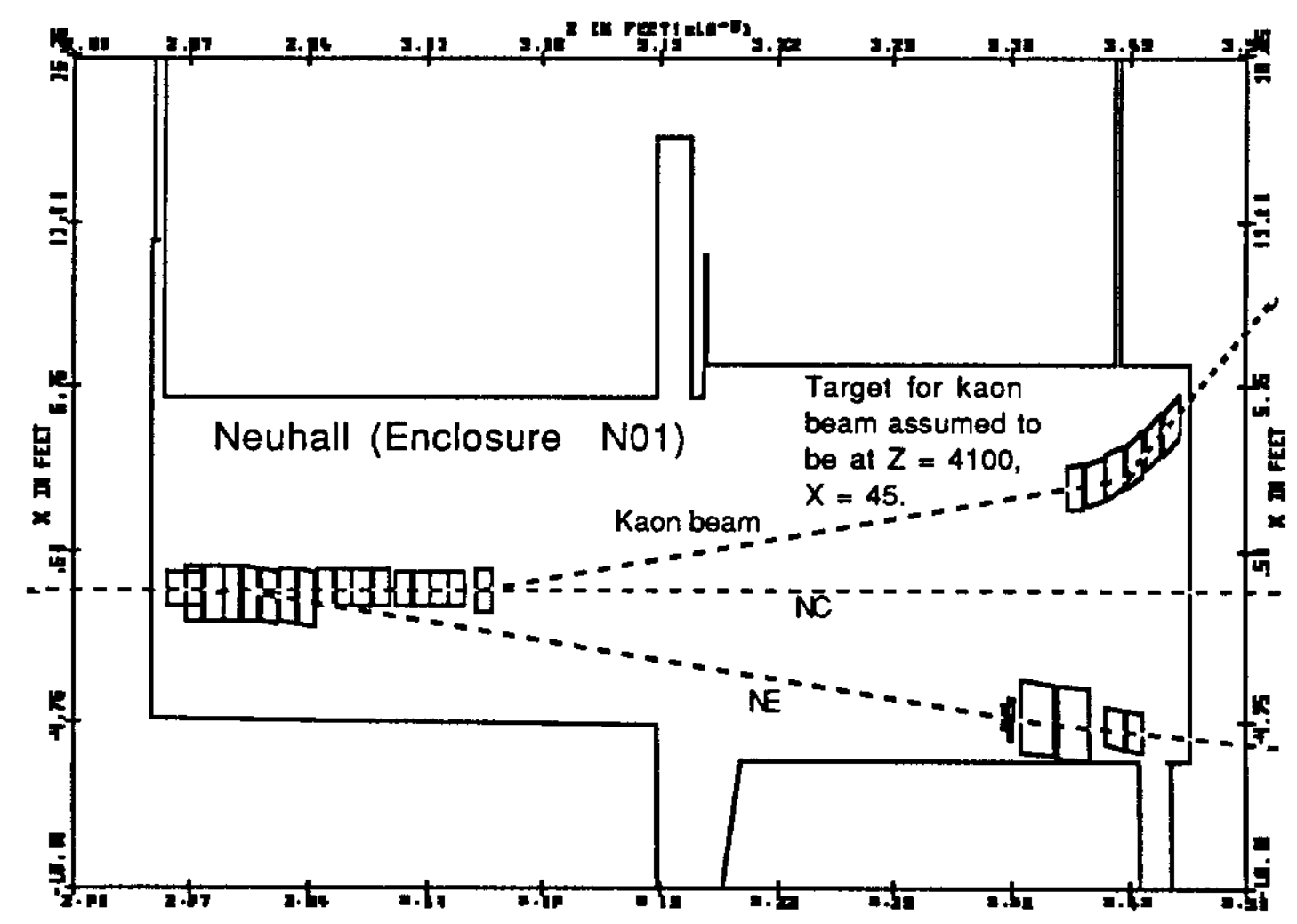

Figure 5.2 
Figure 5.3 shows the $120 \mathrm{GeV}$ beam sizes given by TRANSPORT starting at the upstream end of Neuhall, $Z=2846.7$ and ending at the kaon target at $Z=4100$. The sizes are half-width at the base with the top (positive scale) showing the horizontal beam size while the bottom (negative scale) shows the vertical beam size.

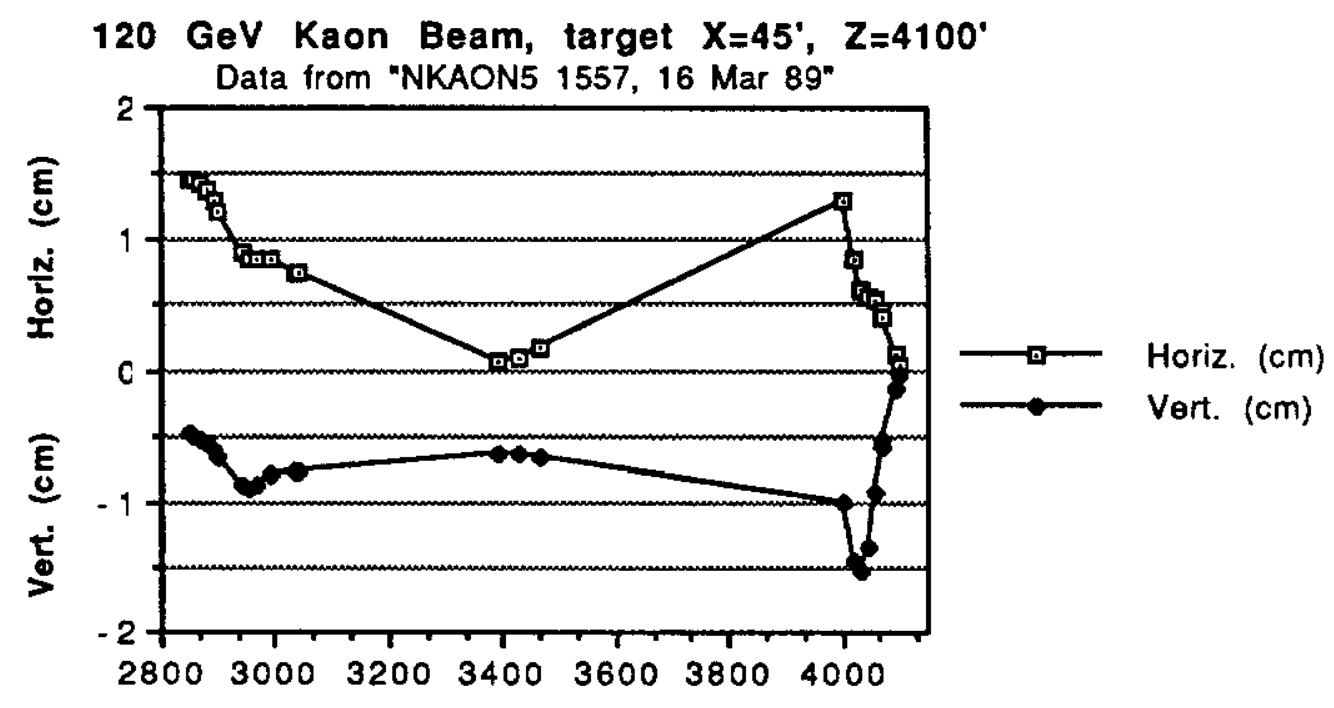

Figure 5.3

The constraint requested by potential users of the Kaon beam was that the size of the beam at the target should be less than 0.75 $\mathrm{mm}$ in radius. Figure 5.4 shows the beam sizes as given by TRANSPORT with expanded resolution in the region adjacent to the Kaon target. The calculated TRANSPORT values for the vertical and horizontal half-widths at the base at $Z=4100$ are $0.37 \mathrm{~mm}$ and 0.46 $\mathrm{mm}$ respectively. 
$120 \mathrm{GeV}$ Kaon Beam, $X=45^{\prime}, Z=4100^{\prime}$

Data from "NKAON5 1557, 16 Mar 89"

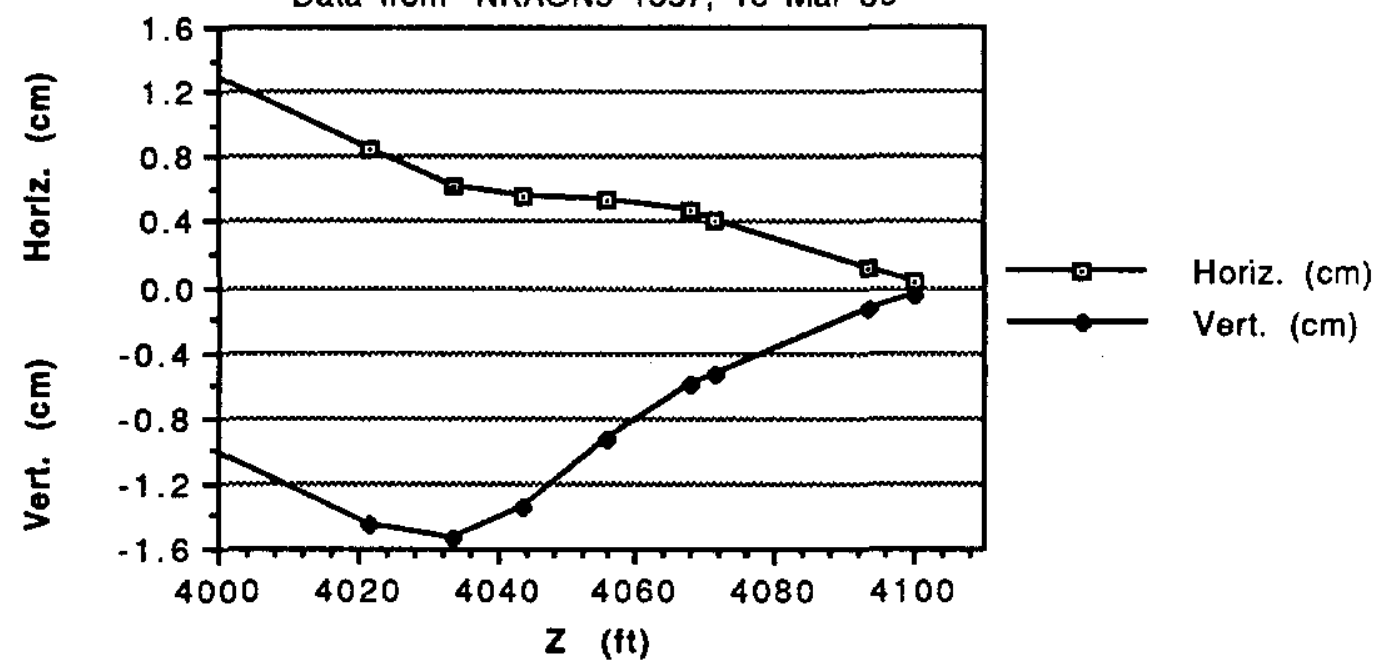

Figure 5.4

Required $120 \mathrm{GeV}$ magnet specifications for elements in the Kaon Beam, starting in Neuhall and ending at the Kaon target, are given in Table 5.1. Downstream of the magnet elements in Neuhall, a pretarget triplet and an invariant angle varying bend (AVB) system are housed in a part of the new building just upstream of the target. All dipoles are assumed to be 5-1.5-120's except for the dipole immediately upstream of the target which is a 5-1.5-240. All quadrupoles are assumed to be 3Q120's. The negative values for the quadrupole gradients are used to indicate defocusing quadrupoles, i.e., defocus in the horizontal plane. Figure 5.5 shows in schematic form pretarget elements of the Kaon beam.

With 3E13 incident protons, we expect 4E9 hz of neutral kaons during a 1 second long slow spill. Neutron rates can be kept at or below the level of kaon rates with appropriate combinations of targeting angle and absorbers. The single track rates from kaon decays within the beam are $2.6 \mathrm{E} 8 \mathrm{hz}$. A forthcoming Technical Memo will describe the neutral kaon beam in detail. 
Table 5.1

$\begin{array}{ccrlr}\begin{array}{c}\text { Magnet } \\ \text { Name }\end{array} & \begin{array}{c}\text { Location } \\ \text { (ft) }\end{array} & \begin{array}{c}\text { Field } \\ \text { (KG or KG/in) }\end{array} & \begin{array}{r}\text { Current } \\ \text { (amps) }\end{array} \\ \text { 1Q1 } & 2867.82 & 0.38 & \mathrm{KG} / \mathrm{in} & 7.32 \\ \text { 1Q2 } & 2955.55 & -0.72 & \mathrm{KG} / \mathrm{in} & 13.81 \\ \text { 1DE } & 2959.87 & 2.53 & \mathrm{KG} & 241.92 \\ \text { 1Q3 } & 2977.66 & 0.32 & \mathrm{KG} / \mathrm{in} & 6.91 \\ \text { 1W } 1 & 3045.25 & 15.00 & \mathrm{KG} & 1648.26 \\ \text { 1W2 } & 3426.80 & 14.61 & \mathrm{KG} & 1569.37 \\ \text { 3Q1-1 } & 4004.68 & 3.21 & \mathrm{KG} / \mathrm{in} & 61.52 \\ \text { 3Q2 } & 4032.63 & -2.76 & \mathrm{KG} / \mathrm{in} & 52.86 \\ \text { 3U } & 4050.61 & 11.00 & \mathrm{KG} & 1039.51 \\ \text { 3Q1-2 } & 4062.57 & 3.21 & \mathrm{KG} / \mathrm{in} & 61.52 \\ \text { 3D } & 4083.53 & 16.50 & \mathrm{KG} & 4271.85\end{array}$

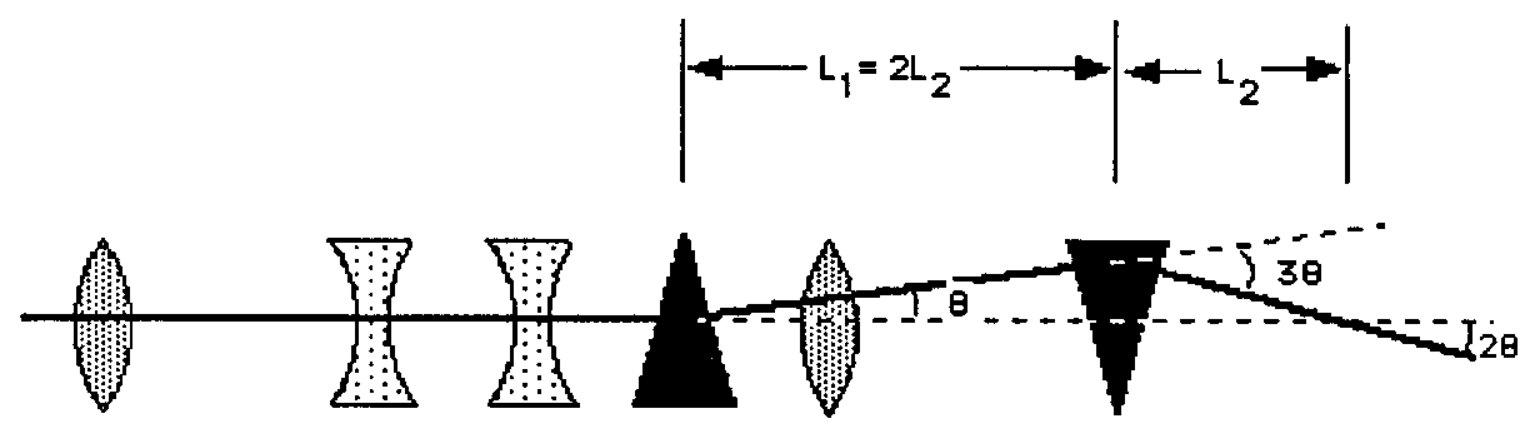

h. focus
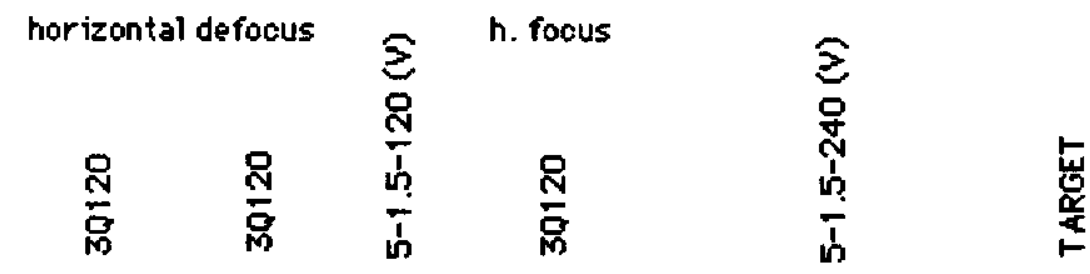

Figure 5.5 


\subsection{Neutrino Oscillation Experiment}

\section{Experiment Placement}

The neutrino oscillation experiment is shown schematically in Figure 5.6. It would consist, in its current version, of a hybrid emulsion detector tucked into the 15 foot bubble chamber magnet; sans bubble chamber of course. The experiment would require the use of a counting house, service building for power supplies and detector gas storage, and a cryogenic refrigerator for the magnet coils.

An optimal location of the experiment might be at the $15^{\prime}$ bubble chamber building, since the desired magnet already exists there. The use of this building, however, would severely restrict the neutrino flux available from the existing neutrino beam, and this restriction would render the experiment uninteresting. An alternative approach was developed that does use Lab $A$, and this will be described as part of a General Neutrino Facility. The model discussed here would move the bubble chamber magnet to a new experimental hall located upstream of Enc. NW6 and $120 \mathrm{~m}$ downstream of the Neutrino beam dump, as shown in Figure 5.7. Size of the new building is estimated as 50' long $X 40^{\prime}$ wide. Construction of the building requires excavation of the neutrino berm and reclamation of steel buried in the berm. This option employs the existing Neutrino area beam line, beam dump and decay pipe. Although this location appears attractive because of the minimal construction required, several problems remain to be solved:

This location for the detector is far removed from any support for cryogenics. It would therefore be necessary to build an adequate building to house a new refrigerator for the bubble chamber magnet. This location would also require that the coils be removed from Lab $A$ and transported to the new location.

An alternative location for the neutrino oscillation experiment could make use of Lab A, utilizing a possible new General Purpose Neutrino Beam, described in the next section. 
Neutrino Oscillation Experiment Plan View

Figure taken from:

Letter of Intent for an Experiment to Improve Limits for

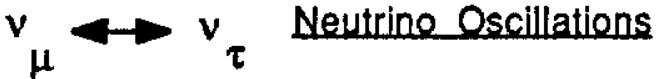

LIpton, Stefanski, Niwa, Frederikson, Reay, Reibel, Sidwell, Stanton, \& Kusumoto

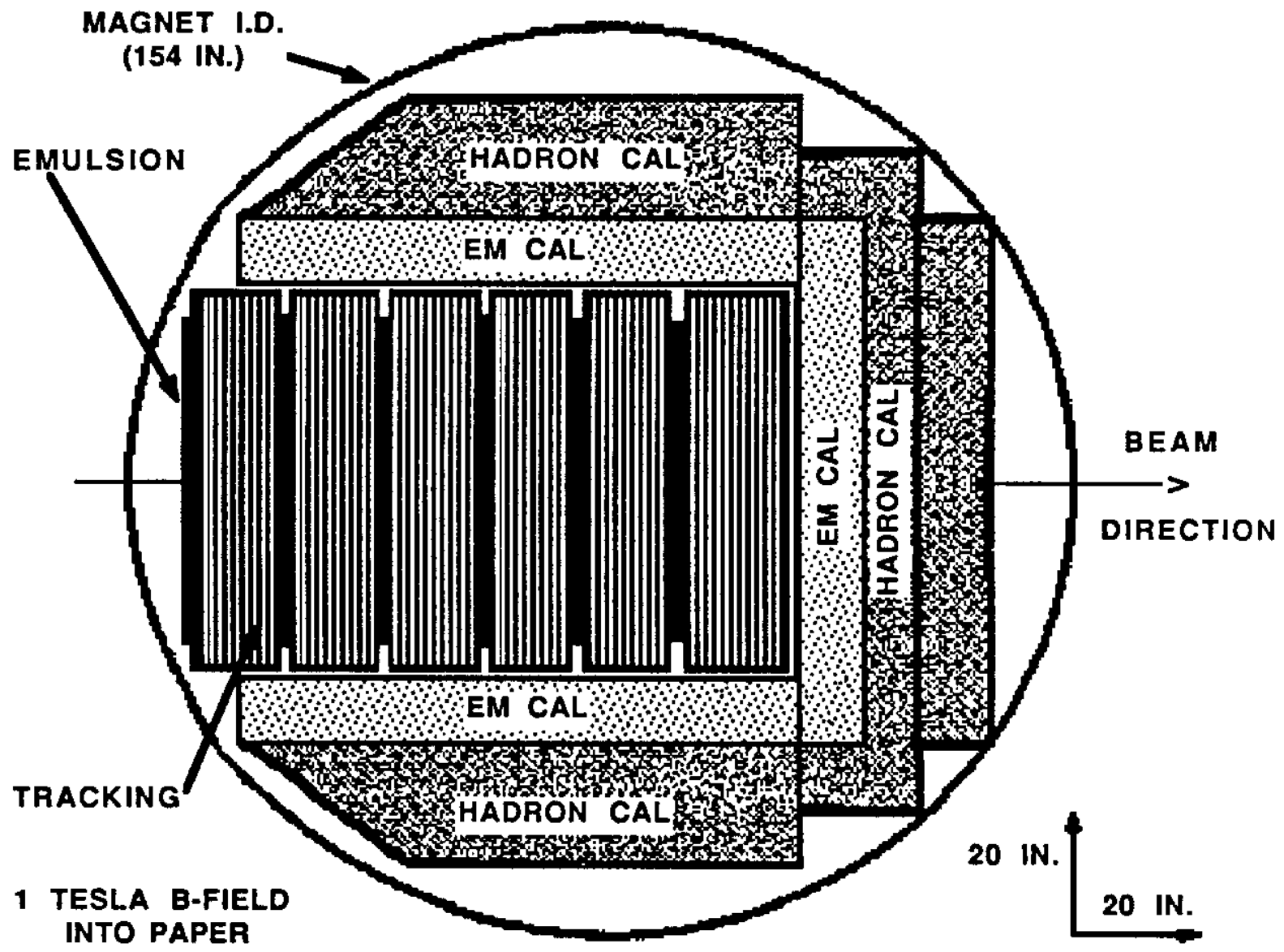

SCINTILLATOR COUNTERS DOWNSTREAM OF ALL EMULSION MODULES AND IN ALL CALORIMETERS NOT SHOWN. MOST MUONS IDENTIFIED BY RANGE AND PULSE HEIGHT IN THE CALORIMETERS. MUON RANGE WALL DOWNSTREAM OF COILS NOT SHOWN.

Figure 5.6 


\section{POSSIBLE 120 - 150 GEV EXPERIMENTAL AREA}

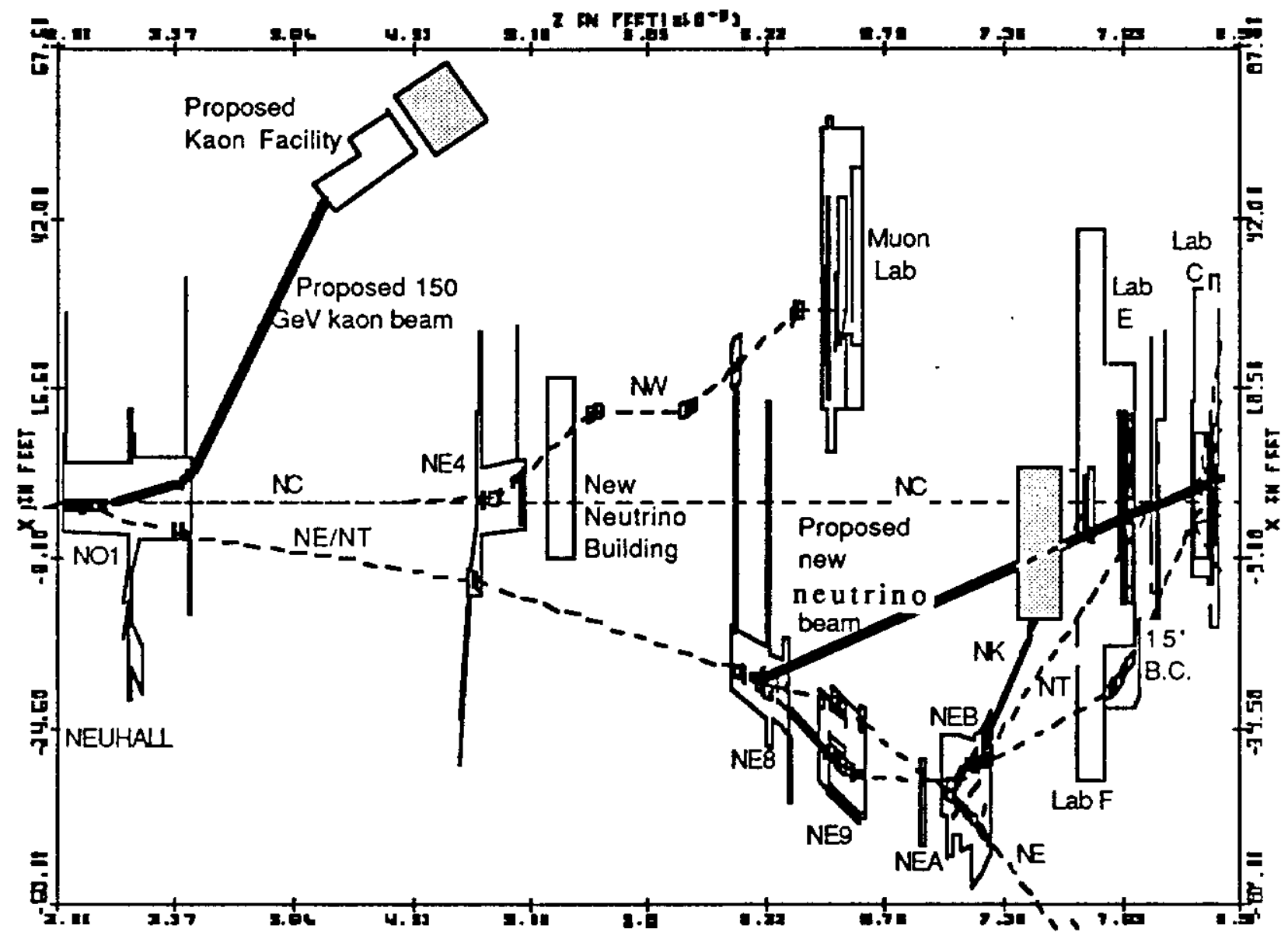

Figure 5.7 


\subsection{General Purpose Neutrino Beam}

The location of this possible new beam is shown also in Figure 5.7. The beam originates in enclosure NE8 which currently is used to house a target station for a $10^{12} \mathrm{ppp}$ beam. This targeting station and shielding would require an upgrade to allow targeting at 3 X1013 ppp, and must provide space for a focusing horn or a lithium lens. The old Muon Lab (NWA) provides space for the pulsed power supply for the focusing system.

Within Enclosure NE8 two of the existing 4-2-240 dipoles would be used to aim the beam toward the $1^{\prime}$ Bubble Chamber. These two dipoles, NT8E2-1 and NT8E2-2, will be located at the same $Z$ 's as their present locations, but must be shifted Westward from their present positions in the NT beam line. With these two dipoles operating at $10.05 \mathrm{KG}$ for $150 \mathrm{GeV}$ beam, the proton beam will be aimed directly at the center of the $15^{\prime}$ Bubble Chamber $(X=-0.667, Z$ $=8071.92$ ) with the beam exiting the downstream wall of Enclosure NE8. The trajectory of the beam downstream of Enclosure NE8 would be similar to the proposed Direct Neutral Lepton Facility beam. This neutrino beam would pass through Labs F, E, B, and C, where neutrino experiments have been conducted in the past.

The beam pipe between NE8 and the beam dump would be oversized in the region of NE9. The distance between this pipe and the NE9 enclosure doesn't allow sufficient steel shielding for personnel to enter the enclosure when the new neutrino beam is operating. Care must be taken to make certain that the beam can never strike the pipe next to the enclosure NE9.

For the neutrino oscillation experiment, the end of the decay pipe must house a beam dump area capable of accepting the High Intensity beam. The beam dump will be located upstream of Enclosure NKC which is presently under construction for Experiment 782. The beam dump itself need only be twenty feet long, including ten feet of aluminum surrounded by steel, followed by a steel plug. The entire mass must be water cooled.

Following the beam dump, sufficient muon shielding must be placed to allow personnel and experiments to operate in the buildings downstream. The dimensions of the steel shield have been 
estimated to be $80 \mathrm{~m}$ long and $4 \mathrm{~m}$ by $4 \mathrm{~m}$ in cross section. The location of the steel would require that the enclosure NKC for the NK beam be removed. This can be done in a cost effective way because the enclosure is built with precast concrete sections that can be reclaimed and reused when the structure is removed.

Because the new neutrino beam line comes into the $15^{\prime}$ Bubble Chamber at an angle of $13.832 \mathrm{mr}$ relative to the old $\mathrm{NC}$ beam, some of the detectors may have to be shifted if they are reused. If desired, the Tohoku Bubble Chamber in Lab $F$ has the capability of being moved into the new beam line, although with some effort. Likewise the neutrino detector at Lab $\mathrm{E}$ has the ability to be shifted into the new beam line.

This beam layout bears a resemblance to the tagged neutrino beam proposed in P-788. To make a tagged neutrino beam certain additions would be required. The target area in enclosure NE8 would have to be modified to include a beam dump and sweeper magnet. Furthermore, the beam-dump area would be converted to an area for the tagging station. This would require a larger enclosure to support an experimental apparatus. 


\subsection{Focusing Systems for a High Flux Neutrino Experiment}

\section{Assumptions}

In this study, the existing Neutrino area target tube has been assumed as the location of the production target and the focusing systems. A one interaction length target has been used in all examples. The production model described in FN-341 (A. J. Malensek, 1982) was used, multiplied by 0.7 , a number chosen to normalize this model to existing data. The current $400 \mathrm{~m}$ decay pipe was used, which has a 0.914 meter radius for the first 54 meters (the target tube), and a 0.457 radius over the remaining length. In order to maximize event rate, the detector has been placed as close to the end of the decay pipe as possible, consistent with ranging out all the muons in the steel shield. This location was chosen to be at $120 \mathrm{~m}$ from the end of the decay pipe. If actually placed at this depth in the berm, some existing steel shielding will have to be removed to make room for the new experimental hall. The detector was assumed to be that of a neutrino oscillation experiment, with 50 liters of emulsion ( 0.2 tons), and a radius of 0.75 meters. Because the tau has a much larger mass than the muon, and thus the tau neutrino cross section is depressed relative to the muon neutrino cross section at lower energies, it was decided to quote event rates for neutrino interactions with a minimum cut on neutrino energy of $10 \mathrm{GeV}$.

\section{Ideal Spectra - Perfect Focus and Bare Target Production}

As a figure of merit with which to compare actual focusing systems, two ideal cases can be described. These are perfect focus, in which all of the mesons produced are directed parallel to the axis of the decay pipe, and bare target, in which no focusing system is employed. These then are the two extremes between which all focused neutrino beams will fall. In reality, the best beam designs fall somewhat short of perfect focus/2. Figure 5.8 shows the 
neutrino event rate $/ \mathrm{GeV} / \mathrm{m}^{2}$ as a function of neutrino energy, for $10 * * 13$ protons on target, in the 0.2 ton detector, for both perfect focus $/ 2$ and bare target production. For the experiment considered, getting a very small number of anti-neutrinos, which provide a major background to the tau oscillation signal, is just as important as getting a large number of neutrinos. Thus, for all systems considered, both neutrino and anti-neutrino event rates will be quoted. Integrated event rates above $10 \mathrm{GeV}$ neutrino energy for $10 * * 13$ protons are:

$\begin{array}{lcc} & v & \bar{v} \\ & & \\ \text { Perfect focus/2: } & 1.36 * 10^{-2} & 3.32 * 10^{-3} \\ \text { Bare target: } & 6.94 * 10^{-4} & 1.03 * 10^{-4}\end{array}$

\section{Horn Focusing Systems}

Two different horn systems have been employed here at Fermilab in past years. One was an elaborate three horn design, constructed as two physical units called Horn \#1 and Horn \#2, connected in series by a high current transmission line. This horn system is described in detail in TM-555 (Frank Nezrick, 1975). The other horn system was a simpler single horn design, built after the first system, to minimize operational difficulties. It is described more fully in TM-824 (J. Grimson and S. Mori, 1978). For this study, the single horn system was run at $140 \mathrm{KA}$. Neutrino event rates for the two-horn system are shown in Figure 5.9. For these horn systems, integrated event rates above $10 \mathrm{GeV}$ neutrino energy for $10 * * 13$ protons are:

Double horns:

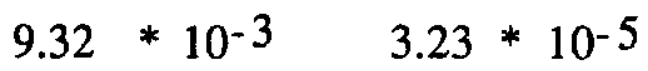

Single horn:

$4.07 * 10^{-3}$

$5.93 * 10^{-5}$ 
The advantages for this experiment of the double horn system, which gives about twice the event rate of the single horn system, are apparent. Preliminary discussions with Research Division mechanical and electrical engineers indicate that many of the problems encountered with the more complicated double horn system can be solved using the techniques learned with the more robust single horn design.

\section{Lithium Lens Focusing Systems}

Another method employed to gather up large numbers of hadrons while defocusing opposite sign particles is a system made up of lithium lenses. A single lithium lens is in operation here at Fermilab as part of the p-bar production facility. These lenses have magnetic fields which grow linearly with lens radius, and thus act like quadrupoles which focus (or defocus) particles in both planes. A single short lens would only focus a small momentum range of secondaries; so to have a wide momentum acceptance, one would need to build a system of multiple lenses, or perhaps to make longer single lenses. This is illustrated in the following table, which compares neutrino event rates per $10 * * 13$ protons in the 0.2 ton detector for a variety of lithium lenses.

$\begin{array}{lcc} & v & - \\ & & v \\ \text { P-bar lens: } & 1.49 * 10^{-3} & 7.00 * 10^{-5} \\ \text { Russian lenses: } & 1.14 * 10^{-2} & 1.24 * 10^{-5} \\ \text { Three p-bar lens: } & 9.18 * 10^{-3} & 3.23 * 10^{-5} \\ 30 \mathrm{~cm} \text { lens: } & 8.32 * 10^{-3} & 3.78 * 10^{-5}\end{array}$


The first lens system is just the Fermilab p-bar lens, which is a single lens, $15 \mathrm{~cm}$ in length, $1 \mathrm{~cm}$ in radius, located $14.5 \mathrm{~cm}$ from the target. The event rate quoted is for an $80 \mathrm{kG} / \mathrm{cm}$ field gradient, the gradient typically run for p-bar production. One can see that this lens is only a factor of two improvement in event rate over bare target production.

A much more elaborate lens system is the "Russian" lens system, which was scaled from a paper on neutrino beams for the UNK accelerator (E. M. Boldyrev, R. A. Rzaev and V. P. Sakharov, 1987, unpublished). It employs 3 separate lenses, all about $15 \mathrm{~cm}$ or so in length, but of various diameters $(0.9 \mathrm{~cm}, 1.7 \mathrm{~cm}$ and $3.0 \mathrm{~cm})$ and field gradients $(80 \mathrm{kG} / \mathrm{cm}, 70 \mathrm{kG} / \mathrm{cm}$ and $40 \mathrm{kG} / \mathrm{cm})$. This system was designed to maximize neutrino event rate while minimizing anti-neutrino background, a job it does remarkably well. The technical problems in constructing lenses with radii greater than $1 \mathrm{~cm}$ in size, and with surface fields exceeding $100 \mathrm{kG}$ need to be noted, however.

Two more modest systems are also shown. The first employs three p-bar style lenses of $15 \mathrm{~cm}$ in length, all running at the same field gradient of $68 \mathrm{kG} / \mathrm{cm}$. The final system is a single $30 \mathrm{~cm}$ long lens, $1 \mathrm{~cm}$ in diameter, operating at $80 \mathrm{kG} / \mathrm{cm}$. Neutrino event rates for the single $30 \mathrm{~cm}$ lens are shown in Figure 5.10 .

One large technical difficulty in choosing a lithium lens for the neutrino focusing system is the fact that to date these lenses have been pulsed with short ( a few micro-second) current pulses. To target $2-3 * 10 * * 13$ protons, much longer beam spill times are needed (typically about a millisecond). A substantial $R$ and $D$ effort would be needed to study whether or not this is feasible for the lithium lenses. 


\section{Discussion}

It should be pointed out that these neutrino event rate calculations did not take into account absorption or additional secondary production in the focusing systems themselves. This could substantially affect the rates quoted for the more massive systems, both for the neutrino signal and the anti-neutrino background rates. In addition, further sources of anti-neutrino backgrounds have not been included, such as $K_{L} \rightarrow \pi \mu v$.

With the large technical problems involved in mounting any of the lithium lens systems, it seems clear that magnetic horns are the preferred choice for a neutrino focusing system. The double horn system, because of its large event rate, is preferred over the simple single horn system.

\section{Other Possibilities}

The CERN wide-band neutrino program is capable of delivering neutrino event rates of 0.13 events/ton for $10 * * 13$ protons on target. (Particle Data Group publication, "Current Experiments in Elementary Particle Physics, 1987). When the different cycle times are factored in, this must be viewed as a competitive program, if resources there were committed to a similar project. 


\section{Neutrino Event Rates}

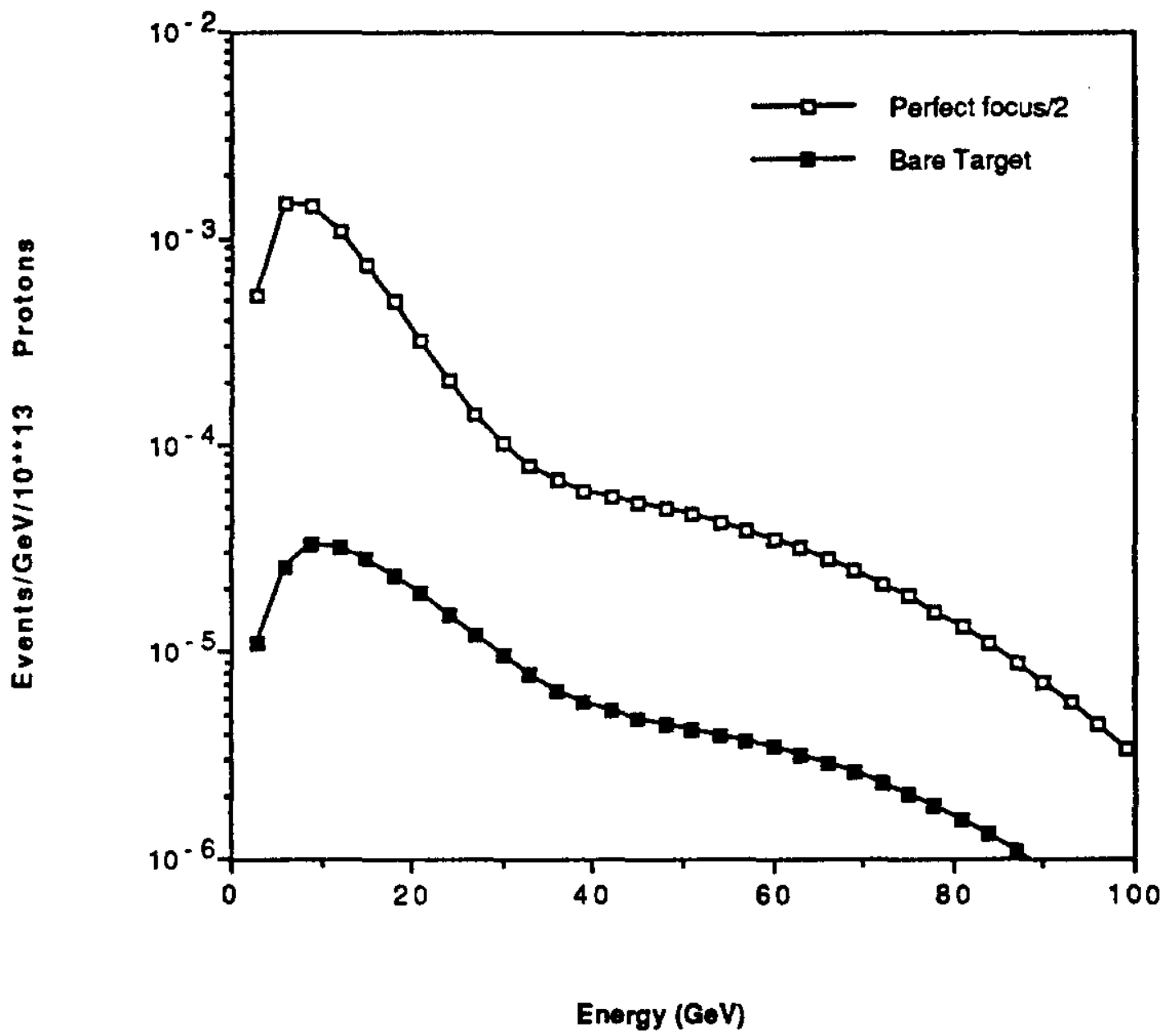

Figure 5.8 


\section{Two Horn System}

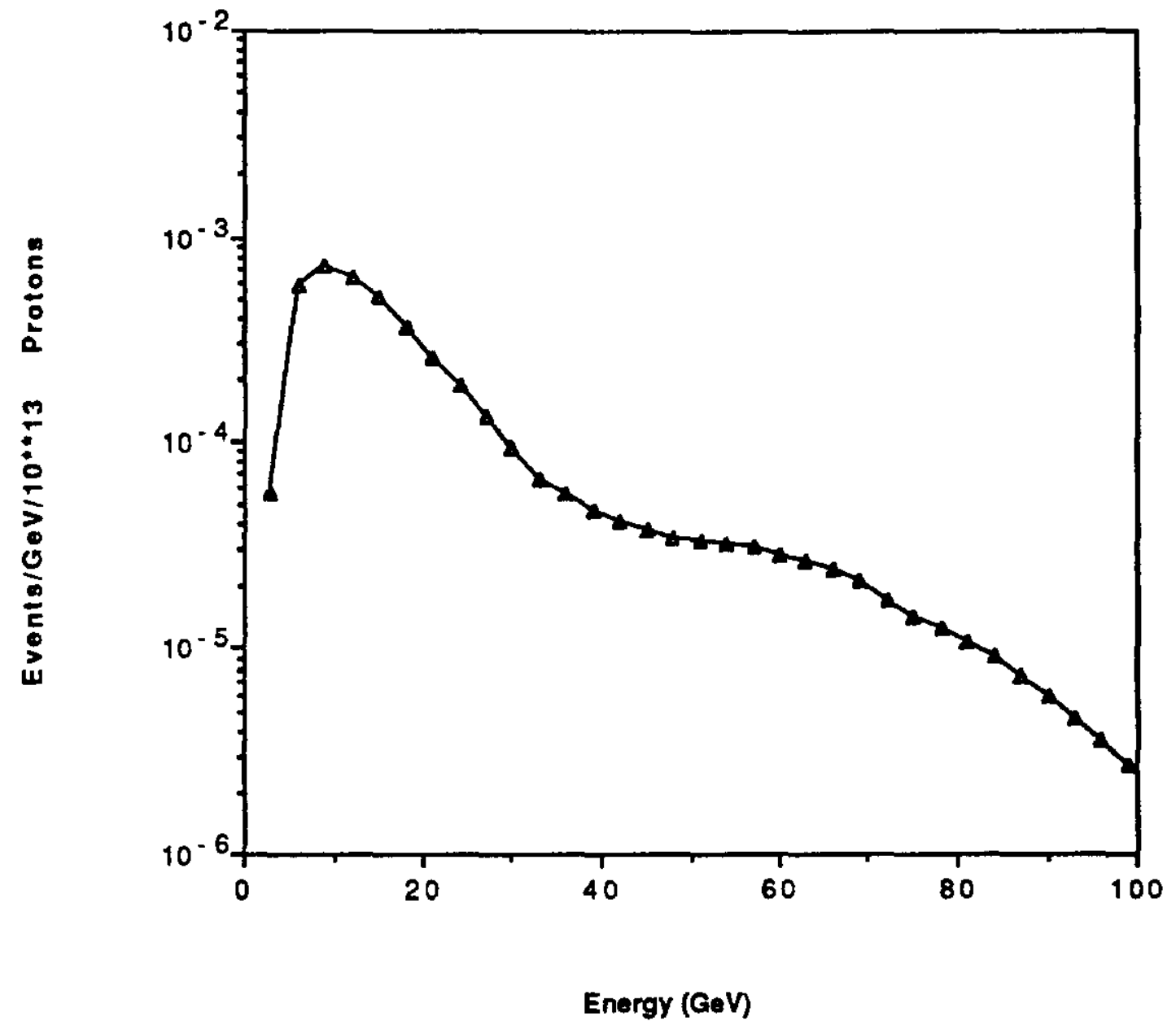

Figure 5.9 


\section{$30 \mathrm{~cm}$ Lithium Lens}

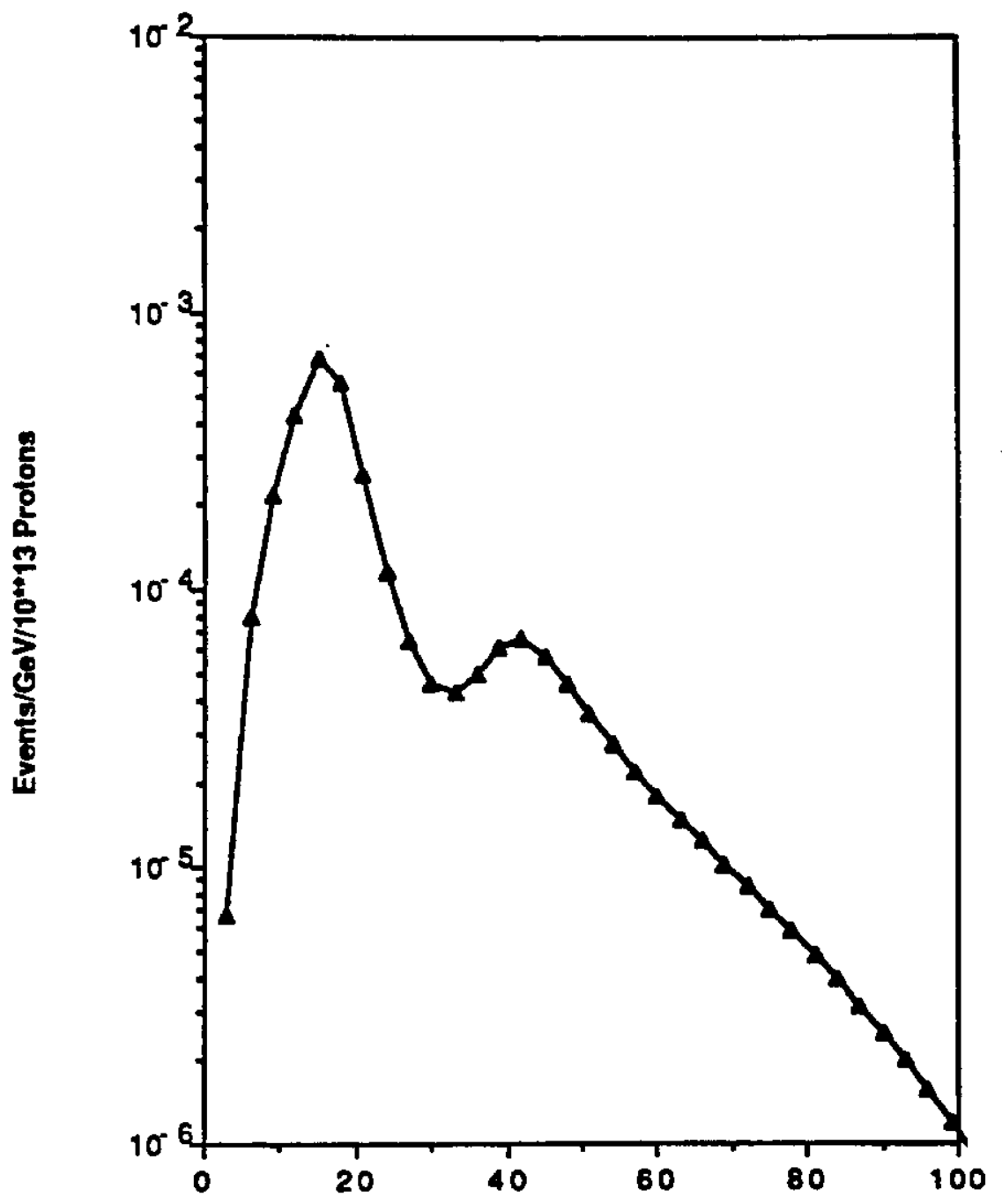

Energy (GoV)

Figure 5.10 


\subsection{Cooling Requirements for High Intensity Target and Beam Dump}

Target and beam dump integrity becomes a cause for serious concern for the beam fluxes possible with extraction from the Main Injector. Cooling requirements for a beryllium target and subsequent beam dump exposed to the full luminosity extracted beam have thus been determined. The computer code CASIM has been used for modeling energy deposition in the target and dump materials.

\section{Targeting Assumptions}

A horizontal and vertical beam size of $\sigma=1 \mathrm{~mm}$ at $150 \mathrm{GeV}$ was assumed with an intensity per pulse of $3 \mathrm{E} 13$ protons. The cycle time was assumed to be 3 seconds. Slow spill duration is taken as 1 second, while fast spill is assumed to be a few milliseconds in duration. The target was taken to be $50 \mathrm{~cm}$ of beryllium having a radius of $2.25 \mathrm{~mm}$. [When $\mathrm{r}=2.25 \sigma, 92 \%$ of the proton beam is contained in a cross-section of diameter $2 r$.] The beam dump used has iron surrounding a copper insert with dimensions 3 inches by 24 inches, in the bend plane of the magnetized dump.

\section{Target Cooling Requirements}

About 450 watts is deposited in the beryllium target for each beam pulse. For slow spill, the diffusion time for the temperature distribution to spread from the targeting center outward to the full beam size is small compared to the spill time of 1 second. Thus an average energy deposition throughout the target can be used: EBAR = $0.035 \mathrm{GeV} / \mathrm{cm}^{3}$.

The one pulse $\Delta \mathrm{T}$ rise is then $50^{\circ} \mathrm{C}$. For radiation and natural convection cooling only, steady state is achieved after roughly 60 pulses, with a temperature of $950^{\circ} \mathrm{C}$. Even though this is below the melting point of beryllium, it is high enough to cause concern. On the other hand, conduction via water cooling is more than adequate. A modest flow rate, such as 1 gallon/minute through the target and target base would produce only a few degrees rise as the water passes through. Forced convection, perpendicular to the target axis and using air with a flow rate of 4 meters/second, gives a steady 
state temperature of $605^{\circ} \mathrm{C}$. A flow rate of 20 meters/second gives $350^{\circ} \mathrm{C}$.

For fast resonant extracted beam with a few milliseconds duration, the dynamic stresses commonly referred to as thermal shock are negligible. Thermal diffusion time is now longer than the spill time, so that peak temperature of the target is concentrated at the center, near the downstream end where the cascade is most developed. The maximum $\Delta \mathrm{T}$ rise for one pulse is about $145^{\circ} \mathrm{C}$. Clearly, water cooling is a necessity for fast spill. Also, heavy targets like $\mathrm{Cu}$ or $\mathrm{W}$ [assuming the same beam size and target size as the $\mathrm{Be}$ ] are inappropriate; the energy deposition in them would be sufficiently large that the melting point is exceeded during one beam pulse.

\section{Dump Cooling Requirements}

About 145 kwatts of power is deposited in the copper and iron of the beam dump for every pulse of $3 \mathrm{E} 13$ protons at $150 \mathrm{GeV}$. Clearly, one or both pieces need to be water cooled. Cooling water is typically available at 80 psi. With a flow rate of 15 gallons/minute, the temperature difference between incoming and outgoing water will be about $30^{\circ} \mathrm{C}$. Thus for an initial temperature of $35^{\circ} \mathrm{C}$, the final water temperature will be $65^{\circ} \mathrm{C}$. To obtain 15 gallons/minute with $80 \mathrm{psi}$ at the header requires parallel water cooling lines for reasonable pipe sizes $(\approx 0.5$ inch ID). If this is done, cooling the copper/iron dump for slow spill presents no unusual difficulites.

However, for fast spill, heating problems could arise if the dump is placed close to the target. To control deposited energy density, the size of the beam at the dump face should have grown to be larger than a few millimeters. For a neutrino experiment, where it is desired to maximize the number of decays, the dump is quite far from the target. Then the beam size is typically on the order of a centimeter. Under these conditions, dumping of the fast spill beam is not a problem. 


\section{Conclusions}

Cooling of a beryllium target (50 $\mathrm{cm}$ by $4.5 \mathrm{~mm}$ diameter) exposed to the full extracted Main Injector beam can be accomplished with forced convection, or with a very small amount of water cooling, for the kaon-type experiment envisioned $(1 \mathrm{sec}$. slow spill). Water cooling is definitely needed for a typical neutrino experiment ( few ms. fast spill). Beryllium or beryllium oxide targets will be reliable, if water cooled; heavy targets, however, will fail for fast spill with the targeted beam size and intensity considered.

The dump needs to be water cooled, probably with parallel lines. In the case of fast spill the beam size on the dump is required to be $\sigma \geq 5 \mathrm{~mm}$. Since the water for both systems will be closed-loop, and relatively small in volume, a heat exchanger will most likely be required to return the $65^{\circ} \mathrm{C}$ water back down to $35^{\circ} \mathrm{C}$. 